\title{
The Hamiltonian dynamics of Hořava gravity
}

\author{
Deniz O. Devecioğlu ${ }^{1, a}$, Mu-In Park ${ }^{2, b}$ \\ ${ }^{1}$ School of Physics, Huazhong University of Science and Technology, Wuhan 430074, Hubei, China \\ ${ }^{2}$ Center for Quantum Spacetime, Sogang University, Seoul 121-742, Republic of Korea
}

Received: 27 January 2020 / Accepted: 10 June 2020 / Published online: 2 July 2020

(c) The Author(s) 2020

\begin{abstract}
We consider the Hamiltonian formulation of Hořava gravity in arbitrary dimensions, which has been proposed as a renormalizable gravity model for quantum gravity without the ghost problem. We study the full constraint analysis of the non-projectable Hořava gravity whose potential, $\mathcal{V}(R)$, is an arbitrary function of the (intrinsic) Ricci scalar $R$ but without the extension terms which depend on the proper acceleration $a_{i}$. We find that there exist generally three distinct cases of this theory, $\mathbf{A}, \mathbf{B}$, and $\mathbf{C}$, depending on (i) whether the Hamiltonian constraint generates new (secondclass) constraints or just fixes the associated Lagrange multipliers, or (ii) whether the IR Lorentz-deformation parameter $\lambda$ is at the conformal point or not. It is found that, for Cases $\mathbf{A}$ and $\mathbf{C}$, the dynamical degrees of freedom are the same as in general relativity, while, for Case $\mathbf{B}$, there is one additional phase-space degree of freedom, representing an extra (odd) scalar graviton mode. This would achieve the dynamical consistency of a restricted model at the fully non-linear level and be a positive result in resolving the long-standing debates about the extra graviton modes of the Hořava gravity. Several exact solutions are also studied as some explicit examples of the new constraints. The structure of the newly obtained, "extended" constraint algebra seems to be generic to Hořava gravity and its general proof would be a challenging problem. Some other challenging problems, which include the path integral quantization and the Dirac bracket quantization are discussed also.
\end{abstract}

\section{Introduction}

11 years ago, Hořava proposed a renormalizable, higherderivative gravity theory, without the ghost problem in the usual tensor graviton modes, which reduces to Einstein gravity in low energy (IR) at the full action level but with improved

\footnotetext{
a e-mail: dodeve@gmail.com

b e-mail: muinpark@gmail.com (corresponding author)
}

high-energy (UV) behaviors, by abandoning the Lorentz symmetry from non-equal-footing treatment of space and time [1,2]. However, due to absence of the full diffeomorphisms (Diff), extra graviton modes can be expected generally and there have been questions about the recovery of general relativity (GR) in IR, and more generally, the consistency of Hořava gravity [3-16].

In this paper, we reconsider the Hamiltonian formulation of the non-projectable Hořava gravity whose potential, $\mathcal{V}(R)$, is an arbitrary function of the (intrinsic) Ricci scalar $R$ in arbitrary dimensions but without the extension terms which depend on the proper acceleration $a_{i}=\partial_{i} N / N$, for simplicity. We study the full constraint analysis and find that there exist generally three distinct cases of this theory, $\mathbf{A}, \mathbf{B}$, and C, depending on (i) whether the Hamiltonian constraint generates new (second-class) constraints, or just fixes the associated Lagrange multipliers, according to Dirac's method [17], or (ii) whether the IR Lorentz-deformation parameter $\lambda$ is at the conformal point, $\lambda=1 / D$, or not. It is found that, for Cases $\mathbf{A}$ and $\mathbf{C}$, the dynamical degrees of freedom are the same as in general relativity, while, for Case $\mathbf{B}$, there is one additional phase-space degree of freedom, representing an extra (odd) scalar graviton mode. This would achieve the dynamical consistency of a restricted model at the fully non-linear level and be a positive result in resolving the long-standing debates about the extra graviton modes of the Hořava gravity.

The organization of the paper is as follows. In Sect. 2, we consider the set-up for the Hamiltonian formulation of the non-projectable Hořava gravity in arbitrary dimensions. In Sect. 3, we consider Dirac's constraint analysis when $\lambda \neq 1 / D$ for the IR Lorentz-deformation parameter $\lambda$ and study two cases, $\mathbf{A}$ and $\mathbf{B}$, depending on whether the Hamiltonian constraint generates new (second-class) constraints or just fixes the associated Lagrange multipliers. In Sect. 4, we consider the third case, $\mathbf{C}$, when $\lambda=1 / D$, which is at a conformal point and generates new (second-class) constraints similar to Case A. We show that the dynamical degrees of 
freedom are the same as in GR for Cases $\mathbf{A}$ and $\mathbf{C}$, while there is one additional phase-space degree of freedom for Case $\mathbf{B}$ which representing an extra (odd) scalar graviton mode. In Sect. 5, we study several exact solutions as some explicit examples of the new constraints. In Sect. 6, we conclude with remarks on several challenging theoretical problems, which include the path integral quantization and the Dirac bracket quantization.

\section{The Hamiltonian formulation in arbitrary dimensions: set-up}

In this section, we consider the Hamiltonian formulation of the non-projectable Hořava gravity in arbitrary dimensions. To this ends, we start by considering the ADM decomposition of the metric [18]

$d s^{2}=-N^{2} d t^{2}+g_{i j}\left(d x^{i}+N^{i} d t\right)\left(d x^{j}+N^{j} d t\right)$

with the arbitrary space-time dependent lapse and shift functions $^{1}, N, N^{i}$, and induced metric $g_{i j}(i, j=1,2, \ldots D)$ on a time-slicing hypersurface $\Sigma_{t}$. Then, the action on a $(D+1)$ dimensional manifold $\mathcal{M}$ with the boundary $\partial \mathcal{M}$ is given by

$$
\begin{aligned}
S= & \int_{\mathcal{M}} d t d^{D} x \sqrt{g} N\left\{\frac{2}{\kappa^{2}}\left(K_{i j} K^{i j}-\lambda K^{2}\right)-\mathcal{V}\left[g_{i j}\right]\right\} \\
& +S_{\partial \mathcal{M}}
\end{aligned}
$$

with an appropriate boundary action ${ }^{2} S_{\partial \mathcal{M}}$ and the potential $\mathcal{V}\left[g_{i j}\right]$, which depends only on the metric $g_{i j}$ and its spatial derivatives $[1,2]^{3}$. Here,

$K_{i j}=\frac{1}{2 N}\left(\dot{g}_{i j}-\nabla_{i} N_{j}-\nabla_{j} N_{i}\right)$

is the extrinsic curvature (the overdot $\left({ }^{*}\right)$ denotes the time derivative) of the hypersurface $\Sigma_{t}$ and $K \equiv g_{i j} K^{i j}$ denotes its trace.

\footnotetext{
${ }^{1}$ For the projectable case, where the lapse function depends only on time coordinate, i.e., $N(t)$, there is no "local" Hamiltonian constraint so that there is no smooth way to recover the usual constraint algebra in GR $[1,6]$. Later, we will comment about other alternative formulation which can provide a smooth limit to the non-projectable case.

2 The explicit form of boundary terms is not essential in this paper and will not be considered in detail. But, due to the similarity to GR or its Lorentz invariant higher-curvature gravity theories, the required boundary terms are also quite close [19], whose IR limit agrees with those of [20,21], for example.

3 The UV Lorentz violation due to higher-spatial-derivative terms in the potential part is originated from the works of Lifshitz [22] in the study of, so called, "Lifshitz field theories". On the other hand, the $I R$ Lorentz violation due to the deformation parameter $\lambda \neq 1$ in the kinetic part was first studied by DeWitt in the study of "canonical quantum gravity" [23]. So, it would be desirable to call the bulk part of action (2) as DeWitt-Horava-Lifshitz (DHL)'s action, more precisely.
}

It is well known that GR is not renormalizable in the conventional way. Its Lorentz invariant higher-curvature modifications have some improvements of UV behaviors but there are more dynamical degrees of freedom than those of GR generally, and the existence of negative kinetic energy modes in the additional degrees of freedom, called ghost modes, is unavoidable due to higher-time derivatives [24-26]. In order to avoid the possible problems of ghost degrees of freedom, we do not simply consider the higher-time-derivative terms, like $K^{4},\left(K_{i j} K^{i j}\right)^{2}$, etc., but only consider the second-order time-derivative terms, like $K_{i j} K^{i j}$ and $K^{2}$, in the kinetic part of the action (2). Whereas, in order to achieve the renormalizable theory with the improved UV behaviors, we consider the higher-spatial-derivative terms, like $R^{2}, R_{i j} R^{i j}$, etc., in the potential part $\mathcal{V}\left[g_{i j}\right]$ with the intrinsic Ricci curvature $R_{i j}$ and its trace $R \equiv g_{i j} R^{i j}$. In order that the theory be power-counting renormalizable, the potential part needs to contain " $2 \times D$ " (spatial) derivatives at least, which is sometimes represented by the dynamical critical exponent, $z=D$ [2]. In order that this construction of a renormalizable action is not spoiled by the mixing of space and time (derivatives) in the general coordinate transformations, we need to further constrain the allowed coordinate transformations into the foliation-preserving diffeomorphisms $\left(\right.$ Diff $\left._{\mathcal{F}}\right)$,

$$
\begin{aligned}
\delta x^{i} & =-\zeta^{i}(t, \mathbf{x}), \delta t=-f(t), \\
\delta g_{i j} & =\partial_{i} \zeta^{k} g_{j k}+\partial_{j} \zeta^{k} g_{i k}+\zeta^{k} \partial_{k} g_{i j}+f \dot{g}_{i j}, \\
\delta N_{i} & =\partial_{i} \zeta^{j} N_{j}+\zeta^{j} \partial_{j} N_{i}+\dot{\zeta}^{j} g_{i j}+f \dot{N}_{i}+\dot{f} N_{i}, \\
\delta N & =\zeta^{j} \partial_{j} N+f \dot{N}+\dot{f} N .
\end{aligned}
$$

In $\operatorname{Diff}_{\mathcal{F}}$, each term in the kinetic part is invariant separately and $\lambda$ can be arbitrary ${ }^{4}$ [23]. If we consider $\lambda=1$ and $\mathcal{V}\left[g_{i j}\right]=-\left(2 / \kappa^{2}\right) R-\Lambda$ as in GR, then there is an "accidental" symmetry enhancement which mixes each term in the action so that the full Diff is recovered [8]. So, there are two sources of the Lorentz violations, either from the higher-spatial-derivative (UV) terms in the potential part or from the deformation of kinetic part with an arbitrary $\lambda$ in IR, generally.

For the potential part, one may consider any function having $2 D$ spatial derivatives for the power-counting renormalizability, but in this paper we consider only the function of Ricci curvature scalar, $R$, i.e., $\mathcal{V}\left[g_{i j}\right] \equiv \mathcal{V}(R)$, for simplicity ${ }^{5}$. Then, the first-order formulation of the action (2) is given by

\footnotetext{
${ }^{4}$ For the case $\lambda=1 / D$, where the theory becomes singular, a separate consideration is needed $[10,21]$. We will consider this case later, in Sect. 4.

5 The terms of $\nabla^{2} R, R_{i j} R^{i j}$, etc. could also produce some other peculiar UV behaviors due to ingenious combinations of terms depending on space-time dimensions, but we will not consider this possibility in this paper.
} 
$S=\int_{\mathcal{M}} d t d^{D} x\left\{\pi^{i j} \dot{g}_{i j}-N \mathcal{H}-N_{i} \mathcal{H}^{i}-\partial_{i} \gamma^{i}\right\}$

with appropriate boundary terms, $\partial_{i} \gamma^{i}$, the conjugate momenta,

$\pi^{i j} \equiv \frac{\delta S}{\delta \dot{g}_{i j}}=\frac{2 \sqrt{g}}{\kappa^{2}}\left(K^{i j}-\lambda K g^{i j}\right)$,

and

$\mathcal{H} \equiv \frac{\kappa^{2}}{2 \sqrt{g}}\left[\pi^{i j} \pi_{i j}-\left(\frac{\lambda}{D \lambda-1}\right) \pi^{2}\right]+\sqrt{g} \mathcal{V}$,

$\mathcal{H}^{i} \equiv-2 \nabla_{j} \pi^{i j}$

where $\pi \equiv g_{i j} \pi^{i j}$. Here, we first consider the case $\lambda \neq 1 / D$ so that $\mathcal{H}$ in (7) and the first-order action (5) are not singular.

The Poisson brackets for the canonical variables are given by

$\left\{g_{i j}(x), \pi^{k l}(y)\right\}=\delta_{i j}^{k l} \delta^{D}(x-y)$

with $\delta_{i j}^{k l} \equiv\left(\delta_{i}^{k} \delta_{j}^{l}+\delta_{i}^{l} \delta_{j}^{k}\right) / 2$.

\section{Constraint analysis à la Dirac $(\lambda \neq 1 / D)$}

The primary constraints of the action (2) are given by

$\Phi^{t} \equiv \pi_{N} \approx 0, \Phi^{i} \equiv \pi^{i} \approx 0$

from the definition of conjugate momenta, $\pi_{N} \equiv \delta S / \delta \dot{N}$ and $\pi^{i} \equiv \delta S / \delta \dot{N}_{i}$. Here, the weak equality ' $\approx$ ' means that the constraint equations are used only after calculating the Poisson brackets.

The preservation of the primary constraints, $\Phi^{\mu} \equiv$ $\left(\Phi^{t}, \Phi^{i}\right)$, i.e., $\dot{\Phi}^{\mu}=\left\{\Phi^{\mu}, H_{C}\right\} \approx 0$, as being required by the consistency of the constraints, with the canonical Hamiltonian,

$H_{C}=\int_{\Sigma_{t}} d^{D} x\left\{N \mathcal{H}+N_{i} \mathcal{H}^{i}\right\}+H_{\mathcal{B}}$

produces the secondary constraints,

$\mathcal{H} \approx 0, \mathcal{H}^{i} \approx 0$.

Here, $H_{\mathcal{B}}$ is an appropriate boundary Hamiltonian, $H_{\mathcal{B}}=$ $\oint_{\mathcal{B}} d^{D} x \hat{n}_{i} \gamma^{i}$, for the intersection $\mathcal{B}$ of an arbitrary time-like boundary ( $\hat{n}_{i}$ is its unit normal) with a time slice $\Sigma_{t}$ so that the total boundaries are $\partial \mathcal{M}=\Sigma_{t_{f}} \cup \Sigma_{t_{i}} \cup \mathcal{B}$. On the other hand, the dynamical equations for $g_{i j}$ and $\pi^{k l}$ are obtained as follows, neglecting boundary contributions (see Appendix A for the details),

$$
\begin{aligned}
\dot{g}_{i j} & =\left\{g_{i j}, H_{C}\right\}=\frac{\delta H_{C}}{\delta \pi^{i j}} \\
& =\left(\frac{\kappa^{2}}{2}\right) \frac{2 N}{\sqrt{g}}\left(\pi_{i j}-\tilde{\lambda} g_{i j} \pi\right)+\nabla_{i} N_{j}+\nabla_{j} N_{i},
\end{aligned}
$$

$$
\begin{aligned}
\dot{\pi}^{i j}= & \left\{\pi^{i j}, H_{C}\right\}=-\frac{\delta H_{C}}{\delta g_{i j}} \\
= & \left(\frac{\kappa^{2}}{2}\right) \frac{N}{\sqrt{g}}\left[\frac{1}{2} g^{i j}\left(\pi_{m n} \pi^{m n}-\tilde{\lambda} \pi^{2}\right)\right. \\
& \left.-2\left(\pi^{i m} \pi_{m}^{j}-\tilde{\lambda} \pi \pi^{i j}\right)\right] \\
& -N \sqrt{g}\left[\frac{1}{2} g^{i j} \mathcal{V}(R)-R^{i j} \mathcal{V}^{\prime}(R)\right] \\
& -\sqrt{g}\left[\nabla^{i} \nabla^{j}\left(N \mathcal{V}^{\prime}(R)\right)-g^{i j} \nabla_{m} \nabla^{m}\left(N \mathcal{V}^{\prime}(R)\right)\right] \\
& +\nabla_{m}\left(N^{m} \pi^{i j}\right)-\left(\nabla_{m} N^{i}\right) \pi^{j m}-\left(\nabla_{m} N^{j}\right) \pi^{i m},
\end{aligned}
$$

where $\tilde{\lambda} \equiv \lambda /(D \lambda-1)$ and ()$^{\prime} \equiv d() / d R$.

With the primary constraints in (10), one can consider the extended Hamiltonian with the Lagrange multipliers $u_{\mu}$,

$H_{E}=H_{C}+\int_{\Sigma_{t}} d^{D} x\left(u_{\mu} \Phi^{\mu}\right)$

from the arbitrariness in the equations of motion, due to the primary constraints.

Then, after tedious computations, we obtain the following constraint algebra (see Appendix A for the details),

$$
\begin{aligned}
\{\mathcal{H}(x), \mathcal{H}(y)\}= & C^{i}(x) \nabla_{i}^{x} \delta^{D}(x-y) \\
& -C^{i}(y) \nabla_{i}^{y} \delta^{D}(x-y), \\
\left\{\mathcal{H}(x), \mathcal{H}_{i}(y)\right\}= & -\mathcal{H}(y) \nabla_{i}^{y} \delta^{D}(x-y), \\
\left\{\mathcal{H}_{i}(x), \mathcal{H}_{j}(y)\right\}= & \mathcal{H}_{i}(y) \nabla_{j}^{x} \delta^{D}(x-y) \\
& +\mathcal{H}_{j}(x) \nabla_{i}^{x} \delta^{D}(x-y),
\end{aligned}
$$

or, for the smeared constraints, $\langle\eta \mathcal{H}\rangle \equiv \int d^{D} x \eta \mathcal{H}$, etc., with the smearing functions $\eta$ and $\eta_{i}$,

$$
\begin{aligned}
\{\langle\eta \mathcal{H}\rangle,\langle\zeta \mathcal{H}\rangle\} & =\left\langle\left(\eta \nabla_{i} \zeta-\zeta \nabla_{i} \eta\right) C^{i}\right\rangle \\
\left\{\langle\eta \mathcal{H}\rangle,\left\langle\zeta^{i} \mathcal{H}_{i}\right\rangle\right\} & =-\left\langle\zeta^{i} \nabla_{i} \eta \mathcal{H}\right\rangle \\
\left\{\left\langle\eta^{i} \mathcal{H}_{i}\right\rangle,\left\langle\zeta^{j} \mathcal{H}_{j}\right\rangle\right\} & =\left\langle\left(\eta^{i} \nabla_{i} \zeta^{j}-\zeta^{i} \nabla_{i} \eta^{j}\right) \mathcal{H}_{j}\right\rangle
\end{aligned}
$$

where $^{6}$

$$
\begin{aligned}
C^{i} & \equiv \sqrt{g} \mathcal{V}^{\prime 2}(R) \nabla_{j}\left(\frac{K^{i j}}{\mathcal{V}^{\prime}(R)}\right) \\
& =\left(\frac{\kappa^{2}}{2}\right) 2 \mathcal{V}^{\prime 2}(R) \nabla_{j}\left(\frac{\pi^{i j}-\widehat{\lambda} g^{i j} \pi}{\mathcal{V}^{\prime}(R)}\right)
\end{aligned}
$$

\footnotetext{
${ }^{6}$ From the Jacobi's identity, one may obtain some non-trivial relations about $C^{i}$. For example, from $\{\{\langle\eta \mathcal{H}\rangle,\langle\zeta \mathcal{H}\rangle\},\langle\rho \mathcal{H}\rangle\}+$ $\{\{\langle\zeta \mathcal{H}\rangle,\langle\rho \mathcal{H}\rangle\},\langle\eta \mathcal{H}\rangle\}+\{\{\langle\rho \mathcal{H}\rangle,\langle\eta \mathcal{H}\rangle\},\langle\zeta \mathcal{H}\rangle\}=0$, one obtains " $\left\langle\left.\left(\eta \nabla_{i} \zeta-\zeta \nabla_{i} \eta\right) \Theta{ }^{i}\right|_{N \rightarrow \rho}\right\rangle+($ cyclic permutations about $\eta, \zeta, \rho)=0$ ", where $\Theta^{i}$ is defined by $\Theta^{i} \equiv\left\{C^{i},\langle N \mathcal{H}\rangle\right\}$ and its explicit form is given by (34), wherein the momentum constraint, $\mathcal{H}_{i} \approx 0$, is imposed.
} 


$$
\begin{aligned}
= & \left(-\frac{\kappa^{2}}{2}\right)\left[\left(\mathcal{H}^{i}+2 \widehat{\lambda} \nabla^{i} \pi\right) \mathcal{V}^{\prime}(R)\right. \\
& \left.+2\left(\pi^{i j}-\widehat{\lambda} g^{i j} \pi\right) \nabla_{j} \mathcal{V}^{\prime}(R)\right]
\end{aligned}
$$

with $\widehat{\lambda} \equiv(\lambda-1) /(D \lambda-1)$. If we consider $-\mathcal{V}(R) \equiv \Lambda+$ $\xi R+\alpha R^{n}$ as a typical example with an arbitrary power $n$, we can obtain

$$
\begin{aligned}
C^{i}= & \left(\frac{\kappa^{2}}{2}\right)\left[\left(\mathcal{H}^{i}+2 \widehat{\lambda} \nabla^{i} \pi\right)\left(\xi+\alpha n R^{n-1}\right)\right. \\
& \left.+2\left(\pi^{i j}-\widehat{\lambda} g^{i j} \pi\right) \alpha n \nabla_{j} R^{n-1}\right] .
\end{aligned}
$$

Note that, when the higher-derivative contributions are absent, i.e., $\alpha=0,(23)$ reduces to $C^{i}=\left(\kappa^{2} / 2\right) \xi \mathcal{H}^{i} \approx 0$ and the Hamiltonian constraint, $\mathcal{H} \approx 0$, becomes the first-class constraint as in GR, for either (i) $\lambda=1$, i.e., $\widehat{\lambda}=0$, or (ii) $\pi \approx 0$, representing the maximal slicing, for an arbitrary $\lambda$ [18]. However, when the higher-derivative terms are present, $C^{i}$ does not vanish generally, so that " the Hamiltonian constraint, $\mathcal{H} \approx 0$, becomes the second-class constraint" even for the maximal slicing, $\pi \approx 0$, due to the genuine dynamical degrees of freedom in $\pi^{i j}$, other than the trace part, $\pi$.

On the other hand, we note that the momentum constraint, $\mathcal{H}_{i} \approx 0$, in (12) has the same canonical form as in GR with no higher-derivative corrections ${ }^{7}$ so that we also have the same constraint algebra for $\mathcal{H}_{i},(18)$ or (21), which representing the spatial Diff generator,

$$
\begin{aligned}
\delta_{\zeta} g_{i j} & =\left\{g_{i j},\left\langle\zeta^{k} \mathcal{H}_{k}\right\rangle\right\}=\frac{\delta\left\langle\zeta^{k} \mathcal{H}_{k}\right\rangle}{\delta \pi^{i j}} \\
& =\nabla_{i} \zeta_{j}+\nabla_{j} \zeta_{j}, \\
\delta_{\zeta} \pi^{i j} & =\left\{\pi^{i j},\left\langle\zeta^{k} \mathcal{H}_{k}\right\rangle\right\}=-\frac{\delta\left\langle\zeta^{k} \mathcal{H}_{k}\right\rangle}{\delta g_{i j}} \\
& =\nabla_{m}\left(\zeta^{m} \pi^{i j}\right)-\left(\nabla_{m} \zeta^{i}\right) \pi^{j m}-\left(\nabla_{m} \zeta^{j}\right) \pi^{i m},
\end{aligned}
$$

as in GR. Moreover, the algebras (17) and (18) show that

$$
\begin{aligned}
\delta_{\zeta} \mathcal{H} & =\left\{\mathcal{H},\left\langle\zeta^{k} \mathcal{H}_{k}\right\rangle\right\}=\nabla_{k}\left(\zeta^{k} \mathcal{H}\right), \\
\delta_{\zeta} \mathcal{H}_{i} & =\left\{\mathcal{H}_{i},\left\langle\zeta^{k} \mathcal{H}_{k}\right\rangle\right\}=\nabla_{k}\left(\zeta^{k} \mathcal{H}_{i}\right)+\left(\nabla_{i} \zeta^{k}\right) \mathcal{H}_{k},
\end{aligned}
$$

which tells that $\mathcal{H}$ and $\mathcal{H}_{i}$ behave as, under the spatial Diff, the scalar and vector densities, respectively, as in GR.

Using the above constraint algebra, one can easily find that the preservation of the secondary constraints give

$$
\begin{aligned}
\dot{\mathcal{H}} & =\left\{\mathcal{H}, H_{E}\right\} \\
& =\frac{1}{N} \nabla_{i}\left(N^{2} C^{i}\right)+\nabla_{i}\left(N^{i} \mathcal{H}\right) \approx \frac{1}{N} \nabla_{i}\left(N^{2} \widetilde{C}^{i}\right),
\end{aligned}
$$

\footnotetext{
7 This is a key observation for the proof of Birkhoff's theorem in Hořava gravity [27], in contrast to other general higher-curvature gravities [25, $26]$. But the IR Lorentz-deformation parameter $\lambda$ enters still in the momentum constraint through the relation (6).
}

$$
\begin{aligned}
\dot{\mathcal{H}}_{i} & =\left\{\mathcal{H}_{i}, H_{E}\right\} \\
& =\mathcal{H} \nabla_{i} N+\nabla_{j}\left(N^{j} \mathcal{H}_{i}\right)+\mathcal{H}_{j} \nabla_{i} N^{j} \approx 0
\end{aligned}
$$

which produces the tertiary constraint,

$\widetilde{\Omega} \equiv \nabla_{i}\left(N^{2} \widetilde{C}^{i}\right) \approx 0$,

where

$$
\begin{aligned}
\widetilde{C}^{i} \equiv & \left.C^{i}\right|_{\mathcal{H}_{i} \approx 0}=\left(\frac{\kappa^{2}}{2}\right)\left[2 \widehat{\lambda} \nabla^{i} \pi\left(\xi+\alpha n R^{n-1}\right)\right. \\
& \left.+2\left(\pi^{i j}-\widehat{\lambda} g^{i j} \pi\right) \alpha n \nabla_{j} R^{n-1}\right]
\end{aligned}
$$

from the preservation of $\mathcal{H} \approx 0$ in (28), excluding the trivial case of $N=0$ for all space-time. Here we note that, in the above computations, there are no contributions from the multiplier terms in $H_{E}$ so that we need to consider further steps of preserving the constraints until the multipliers are determined.

Now, one more step of preserving the new tertiary constraint, $\widetilde{\Omega} \approx 0$, gives

$$
\begin{aligned}
\dot{\widetilde{\Omega}} & =\left\{\widetilde{\Omega}, H_{E}\right\} \\
& =\left\{\widetilde{\Omega}, H_{C}\right\}+2 \widetilde{\Omega}\left(\frac{u_{t}}{N}\right)+2 \widetilde{C}^{i} N^{2} \nabla_{i}\left(\frac{u_{t}}{N}\right) \\
& \approx\left\{\widetilde{\Omega}, H_{C}\right\}+2 \widetilde{C}^{i} N^{2} \nabla_{i}\left(\frac{u_{t}}{N}\right) \approx 0 .
\end{aligned}
$$

Then, there are two different constraint systems, with different subsequent procedures, depending on whether $\widetilde{C}^{i}=0$ or $\widetilde{C}^{i} \neq 0$.

A. Case $\widetilde{C}^{i}=0$ : In this case ${ }^{8}$, (32) does not determine the multiplier $u_{t}$ but reduces to

$$
\begin{aligned}
\dot{\widetilde{\Omega}} & \approx\left\{\widetilde{\Omega}, H_{C}\right\} \\
& \approx \nabla_{i}\left(N^{2} \widetilde{\Theta}^{i}\right) \equiv \widetilde{\Xi} \approx 0
\end{aligned}
$$

for preserving the tertiary constraint $\widetilde{\Omega}$ with

$$
\begin{aligned}
\widetilde{\Theta}^{i} \equiv & \left\{\widetilde{C}^{i},\langle N \mathcal{H}\rangle\right\} \\
= & -\left(\frac{\kappa^{2}}{2}\right)^{2} \frac{2 \widehat{\lambda}}{(\lambda D-1) \sqrt{g}} \mathcal{V}^{\prime}(R)\left\{\left[(2 \lambda+1) g^{i j} \pi\right.\right. \\
& \left.\left.-2(\lambda D-1) \pi^{i j}\right] N \nabla_{j} \pi+\pi^{2} \nabla^{i} N\right\} \\
& -\left(\frac{\kappa^{2}}{2}\right) 2 \widehat{\lambda}(D-1) \sqrt{g} \mathcal{V}^{\prime}(R) \nabla^{i}[(\xi R \\
& \left.\left.+\frac{\Lambda D}{D-1}+\frac{D-n}{D-1} \alpha R^{n}\right) N+\nabla^{2}\left(N \mathcal{V}^{\prime}\right)\right]
\end{aligned}
$$

${ }^{8}$ Here, the condition $\widetilde{C}^{i}=0$ does not necessarily mean the constraint equation, which is stronger than the original constraint (30). Actually, the constraint (30) implies that all the components of $\widetilde{C}^{i}$ are not independent so that the condition $\widetilde{C}^{i}=0$ may be subject to more fundamental conditions or constraints. 


$$
\begin{aligned}
& -\left(\frac{\kappa^{2}}{2}\right)\left[2 \widehat{\lambda} \nabla_{i} \pi\left\{\mathcal{V}^{\prime}(R),\langle N \mathcal{H}\rangle\right\}\right. \\
& \left.+\left\{2\left(\pi^{i j}-\widehat{\lambda} g^{i j} \pi\right) \nabla_{j} \mathcal{V}^{\prime}(R),\langle N \mathcal{H}\rangle\right\}\right],
\end{aligned}
$$

where $-\mathcal{V}^{\prime}(R) \equiv\left(\xi+\alpha n R^{n-1}\right)$. Here, the higher-derivative contributions come, either from the constraint (30), $\widetilde{\Omega}$, i.e., $\widetilde{C}^{i}$ in (31), or from the Hamiltonian constraint $\mathcal{H}$ in (7) and (11). One can compute the explicit forms of the higher-derivative contributions with the help of (13) and (14) (see Appendix B for some more details; see also [15] for the case of $n=2$ ) but, due to its messy expression, we will not consider the explicit forms in the analysis below unless it is crucial.

Then, one more time-evolution of the new constraint, $\widetilde{\Xi} \approx$ 0 , will read,

$$
\begin{aligned}
\dot{\Xi} & =\left\{\widetilde{\Xi}, H_{E}\right\} \\
& =\left\{\widetilde{\Xi}, H_{C}\right\}+\nabla_{i}\left(\left.N^{2} \widetilde{\Theta}^{i}\right|_{N \rightarrow u_{t}}+2 N u_{t} \widetilde{\Theta}^{i}\right) \approx 0 .
\end{aligned}
$$

After a long computation, we obtain (see also Appendix B for the details) $)^{9}$

$$
\begin{aligned}
\nabla_{i} & {\left[\left.N^{2} \widetilde{\Theta}^{i}\right|_{N \rightarrow\left(u_{t}-N_{i} \nabla^{i} N\right)}+2\left(u_{t}-N_{i} \nabla^{i} N\right) N \widetilde{\Theta}^{i}\right] } \\
\approx & -\left\{\widetilde{\Xi}, \bar{H}_{C}\right\}=\left(\frac{\kappa^{2}}{2}\right)^{2} 4 \xi \widehat{\lambda}(D-1) \nabla_{i} \\
& \times\left\{N ^ { 2 } \left[2 N \pi^{i j} \nabla_{j}\left[\left(\xi R+\frac{\Lambda D}{D-1}\right) N-\xi \nabla^{2} N\right]\right.\right. \\
& \left.\left.+\xi \nabla^{i}\left[\pi^{j k}\left(R_{j k} N^{2}-2 N \nabla_{j} \nabla_{k} N-\nabla_{j} N \nabla_{k} N\right)\right]\right]\right\} \\
& -\nabla_{j}\left(N^{j} \widetilde{\Xi}\right) \\
& +\left(\pi, \nabla_{i} \pi-\right.\text { dependent terms) } \\
& + \text { (higher-derivative contributions). }
\end{aligned}
$$

Here, it is important to note that the multipliers, $u_{t}, \nabla^{i} u_{t}$, etc., have generally non-vanishing coefficients in the left-hand side so that (36) may provide the equation for determining the multiplier $u_{t}$. However, the similar equation for $u_{i}$ does not exist and $u_{i}$ is still undetermined but this is just a reflection of the first-class nature of the constraint, $\pi^{i} \approx 0$, in (10). This would now complete the Dirac's procedure for finding the complete set of constraints, though we would not try to solve for the explicit solution of the multiplier $u_{t}$. Then, the full set of constraints are given by $\chi_{A} \equiv\left(\pi_{N}, \mathcal{H}, \widetilde{\Omega}, \widetilde{\Xi}\right) \approx$ $0, \Gamma_{B} \equiv\left(\pi^{i}, \mathcal{H}_{i}\right) \approx 0$. Here, the constraints $\chi_{A} \approx 0$ are the

\footnotetext{
${ }^{9}$ For convenience, we consider the modified momentum constraint [28], $\overline{\mathcal{H}}^{i} \equiv \mathcal{H}^{i}+\pi_{N} \nabla^{i} N$, by redefining the multiplier, $u_{t} \rightarrow u_{t}-$ $N_{i} \nabla^{i} N$ in (15) so that $H_{E}=\bar{H}_{C}+\left\langle\left(u_{t}-N_{i} \nabla^{i} N\right) \pi_{N}+u_{i} \pi^{i}\right\rangle$, $\bar{H}_{C} \equiv\left\langle N \mathcal{H}+N_{i} \overline{\mathcal{H}}^{i}\right\rangle$ and $\left\{\Xi,\left\langle N_{j} \mathcal{H}^{j}\right\rangle\right\}=\nabla_{j}\left(N^{j} \widetilde{\Xi}\right)$. In this way, one can compactly collect all $N_{i}$-dependent terms in the left hand side, up to the weakly vanishing term, $\nabla_{j}\left(N^{j} \widetilde{\Xi}\right) \approx 0$. Interestingly, this modified constraint $\overline{\mathcal{H}}^{i}$ satisfies the same constraint algebra (16)-(18) or (19)-(21).
}

second-class constraints with the constraint algebra,

$$
\begin{aligned}
\left\{\pi_{N}(x), \mathcal{H}(y)\right\}= & 0, \\
\left\{\pi_{N}(x), \widetilde{\Omega}(y)\right\}= & -2 \nabla_{i}^{y}\left(N \widetilde{C}^{i}(y) \delta^{D}(x-y)\right) \approx 0, \\
\left\{\pi_{N}(x), \widetilde{\Xi}(y)\right\}= & \Delta(x-y), \\
\{\mathcal{H}(x), \mathcal{H}(y)\}= & C^{i}(x) \nabla_{i}^{x} \delta^{D}(x-y) \\
& -C^{i}(y) \nabla_{i}^{y} \delta^{D}(x-y) \approx 0, \\
\{\mathcal{H}(x), \widetilde{\Omega}(y)\} \approx & \left\{\pi_{N}(x), \widetilde{\Xi}(y)\right\}, \text { etc. },
\end{aligned}
$$

whose determinant, $\operatorname{det}\left\{\chi_{A}, \chi_{B}\right\}$, is generally non-vanishing,

$$
\begin{aligned}
& \operatorname{det}\left\{\chi_{A}(x), \chi_{B}(y)\right\} \\
& \quad \approx(\operatorname{det}\{\mathcal{H}(x), \widetilde{\Omega}(y)\})^{2}(\operatorname{det}\{\widetilde{\Omega}(x), \mathcal{H}(y)\})^{2} \\
& \approx(\operatorname{det}[\Delta(x-y) \Delta(y-x)])^{2},
\end{aligned}
$$

where

$$
\begin{aligned}
\Delta(x-y) \equiv & -\nabla_{i}^{y}\left[2 N \widetilde{\Theta}^{i}(y) \delta^{D}(x-y)\right. \\
& \left.+N^{2}(y)\left(\frac{\delta \widetilde{\Theta}^{i}(y)}{\delta N(x)}\right)\right] \\
= & -2 \widetilde{\Xi} \delta^{D}(x-y)-2 N^{2} \widetilde{\Theta}^{i}(x) \nabla_{i}^{x}\left(\frac{\delta^{D}(x-y)}{N(x)}\right) \\
& -\nabla_{i}^{y}\left[N^{2}(y)\left(\frac{\delta \widetilde{\Theta}^{i}(y)}{\delta N(x)}\right)\right]
\end{aligned}
$$

with

$$
\begin{aligned}
& \left(\frac{\delta \widetilde{\Theta}^{i}(y)}{\delta N(x)}\right)=-\left(\frac{\kappa^{2}}{2}\right)^{2} \frac{2 \widehat{\lambda} \mathcal{V}^{\prime}}{(\lambda D-1) \sqrt{g}}\left\{\left[(2 \lambda+1) g^{i j} \pi\right.\right. \\
& \left.\left.-2(\lambda D-1) \pi^{i j}\right]\left(\nabla_{j} \pi(y)+\pi^{2} \nabla_{y}^{i}\right) \delta^{D}(x-y)\right\} \\
& -\left(\frac{\kappa^{2}}{2}\right) 2 \widehat{\lambda}(D-1) \sqrt{g} \mathcal{V}^{\prime} \nabla_{y}^{i}\left[\left(\xi R+\frac{\Lambda D}{D-1}\right.\right. \\
& \left.\left.\quad+\frac{D-n}{D-1} \alpha R^{n}\right) \delta^{D}(x-y)+\nabla^{2}\left(\mathcal{V}^{\prime} \delta^{D}(x-y)\right)\right] \\
& -\left(\frac{\kappa^{2}}{2}\right)\left[2 \widehat{\lambda} \nabla_{i} \pi\left\{\mathcal{V}^{\prime}(R)(y), \mathcal{H}(x)\right\}\right. \\
& \left.+\left\{2\left(\pi^{i j}-\widehat{\lambda} g^{i j} \pi\right) \nabla_{j} \mathcal{V}^{\prime}(R)(y), \mathcal{H}(x)\right\}\right] .
\end{aligned}
$$

On the other hand, the constraints, $\Gamma_{A} \equiv\left(\pi^{i}, \mathcal{H}_{i}\right) \approx 0$, are the first-class constraints with the vanishing determinant, $\operatorname{det}\left(\left\{\Gamma_{A}, \Gamma_{B}\right\}\right)=0$. Then, the resulting number of dynamical degrees of freedom in the "configuration" space is given by

$$
\begin{aligned}
s & =\frac{1}{2}\left(P-2 N_{1}-N_{2}\right) \\
& =\frac{1}{2}[(D+1)(D+2)-2 \times 2 D-“ 4 ”] \\
& =\frac{1}{2}(D+1)(D-2),
\end{aligned}
$$


where $P=(D+1)(D+2)$ is the number of canonical variables in the "phase" space $\left(N, \pi_{N}, N_{i}, \pi^{i}, g_{i j}, \pi_{i j}\right), N_{1}=$ $2 D$ is the number of the first-class constraints $\left(\pi^{i}, \mathcal{H}_{i}\right) \approx 0$, and $N_{2}=$ "4" is the number of the second-class constraints, $\left(\pi_{N}, \mathcal{H}, \widetilde{\Omega}, \widetilde{\Xi}\right) \approx 0$. Note that, for Case $\mathbf{A}$, the dynamical degrees of freedom are the same as that of GR (in arbitrary dimensions) though the constraint structure is different: ${ }^{10}$ Actually, in GR, i.e., $\widehat{\lambda}=0, \alpha=0$ or the $\lambda$ deformed GR ( $\lambda R$ model) with the condition, $\pi=0$, we have $N_{1}=2(D+1), N_{2}=0$ so that the 2 first-class constraints, $\left(\pi_{N}, \mathcal{H}\right) \approx 0$, in GR or $\lambda$-deformed GR, transform into the 4 second-class constraints, $\left(\pi_{N}, \mathcal{H}, \widetilde{\Omega}, \widetilde{\Xi}\right) \approx 0$, in the Case $\mathbf{A}$ of full Hořava gravity, with maintaining the same dynamical degrees of freedom $s$. This completes the previous linear analysis in $[10,33]$, but now at the "fully non-linear" level. (cf. $[14,27])$.

B. Case $\widetilde{C}^{i} \neq 0$ : This is the more generic case where the conjugate momenta $\pi^{i j}$ and the (scalar) curvature $R$ are arbitrary, with the generic higher-derivative potential, $\mathcal{V}(R)$. In this case, (32) does not yield new constraints but determines the multiplier $u^{t}$ generally ${ }^{11}$ so that the Dirac's procedure may be completed, without further iterations. Then, in contrast to Case $\mathbf{A}$, there are the second-class constraints, $\tilde{\chi}_{A} \equiv\left(\pi_{N}, \mathcal{H}, \Omega\right) \approx 0$, whose determinant $\operatorname{det}\left(\left\{\tilde{\chi}_{A}, \tilde{\chi}_{B}\right\}\right)$ is non-vanishing, generally,

$$
\begin{aligned}
\operatorname{det} & \left\{\tilde{\chi}_{A}(x), \tilde{\chi}_{B}(y)\right\} \\
= & -\operatorname{det}\left\{\pi_{N}(x), \widetilde{\Omega}(y)\right\} \\
& \times \operatorname{det}\{\mathcal{H}(x), \mathcal{H}(y)\} \operatorname{det}\left\{\widetilde{\Omega}(x), \pi_{N}(y)\right\} \\
\approx & 4 \operatorname{det}\left(N \widetilde{C}^{j}(y) \nabla_{j}^{y} \delta^{D}(x-y)\right) \\
& \times \operatorname{det}\left(N \widetilde{C}^{k}(x) \nabla_{k}^{x} \delta^{D}(x-y)\right) \operatorname{det}\left(C^{i}(x) \nabla_{i}^{x} \delta^{D}(x-y)\right)-(x \leftrightarrow y),
\end{aligned}
$$

whereas the first-class constraints, $\Gamma_{A} \equiv\left(\pi^{i}, \mathcal{H}_{i}\right)$, are the same as in Case A. Hence, the resulting number of dynamical degrees of freedom is

$$
\begin{aligned}
s & =\frac{1}{2}[(D+1)(D+2)-2 \times 2 D-“ 3 ”] \\
& =\frac{1}{2}(D+1)(D-2)+\frac{1}{2},
\end{aligned}
$$

with $N_{1}=2 D$ and $N_{2}=$ " 3 ", which shows one extra degree of freedom in phase space, in addition to the usual $(D+$ 1) $(D-2)$ graviton (transverse traceless) modes in arbitrary $(D+1)$-dimensions. ${ }^{12}$ In particular, in $(2+1)$-dimensions,

\footnotetext{
10 Recent constructions of, so called, "minimally-modified" gravity theories [29-32] may correspond to this case also.

11 For some detailed discussion about the determination of the multiplier $u^{t}$, see [15] (see also [13] for an earlier discussion).

12 Here, we do not consider the extension terms which depend on the proper acceleration, $a_{i}=\partial_{i} N / N$, for simplicity. If we include these terms, in addition to the standard action (2) $[1,2]$, the extra modes have "two" phase space degrees of freedom, like the ordinary scalar fields
}

the extra mode is the only dynamical degree of freedom. This result supports the previous case-by-case results $[15,27]$ but in a more generic set-up with arbitrary dimensions and cosmological constant.

The usual increase of dynamical degrees of freedom with higher-time derivatives is the result of the competition between the increased canonical momenta for the higher-time derivative fields and their increased, associated new constraints $[25,26]$. However, for Hořava gravity, there are no increased canonical momenta but exist only the increased second-class constraints: For Case $\mathbf{A}$, the increased second-class constraints are enough to preserve the dynamical degrees of freedom of GR, whereas for Case $\mathbf{B}$, they are not enough and one extra degree of freedom persists in the phase-space.

\section{Constraint analysis at the conformal point, $\lambda=1 / D$ : Case C}

The kinetic part of Hořava action (2) can be written formally as,

$S_{K} \equiv\left(\frac{2}{\kappa^{2}}\right) \int d t d^{D} x \sqrt{g} N\left(K_{i j} G^{i j k l} K_{k l}\right)$,

where $G^{i j k l} \equiv \delta^{i j k l}-\lambda g^{i j} g^{k l}$ is the (generalized) DeWitt metric $[1,2,23]^{13}$. In the previous sections, we have considered the case, $\lambda \neq 1 / D$, so that the DeWitt metric is not degenerated. Then the first-order action (5) can be obtained by considering the Legendre transformation of $S_{K}$,

$S_{K}=\left(\frac{\kappa^{2}}{2}\right) \int d t d^{D} \times \frac{N}{2 \sqrt{g}}\left(\pi^{i j} \mathcal{G}_{i j k l} \pi^{k l}\right)$,

with the conjugate momenta, $\pi^{i j}=\left(2 \sqrt{g} / \kappa^{2}\right) G^{i j k l} K_{k l}$, and the inverse DeWitt metric, $\mathcal{G}_{i j k l}=\delta_{i j k l}-\lambda g_{i j} g_{k l} /(D \lambda-1)$, satisfying $G^{i j m n} \mathcal{G}_{m n k l}=\mathcal{G}_{k l m n} G^{m n i j}=\delta_{k l}^{i j}[1,2]$.

On the other hand, for $\lambda=1 / D$, the DeWitt metric is degenerated and we need to project out the nondegenerate parts only when considering the appropriate inverse of the DeWitt metric. Actually, using the fact that $\left.\widehat{G}^{i j k l} \equiv G^{i j k l}\right|_{\lambda=1 / D}$ has a null eigenvector $g_{i j}$,

$\widehat{G}^{i j k l} g_{i j}=0$,

it is easy to see that its inverse, $\widehat{\mathcal{G}}_{i j k l}$, is given by

$$
\widehat{\mathcal{G}}_{i j k l}=\delta_{i j k l}-\frac{1}{D} g_{i j} g_{k l},
$$

$[7,20,21,34]$, and this may become another different case, say, Case D. Actually, this corresponds to an alternative formulation of the projectable case but now a smooth limit to the non-projectable case exists [7].

${ }^{13} \lambda_{\text {Horava }}=-\lambda_{\text {DeWitt }} / 2$. 
$\widehat{\mathcal{G}}_{i j k l} g^{i j}=0, \widehat{\mathcal{G}}_{i j m n} \widehat{G}^{m n k l}=\widetilde{\delta}_{i j}^{k l}$

with the (projected) Kronecker-delta, $\widehat{\delta}_{i j}^{k l}=\delta_{i j}^{k l}-g_{i j} g^{k l} / D$, satisfying $\widehat{\delta}_{i j}^{k l} g^{i j}=\widehat{\delta}_{i j}^{k l} g_{k l}=0$ [10]. (See also [25,26] for the corresponding analyses in Lorentz invariant higher-curvature gravities.)

In the Hamiltonian formulation, the existence of a null eigenvector in $g_{i j}$ is reflected in the primary constraint,

$\chi \equiv \widehat{\pi}_{i}^{i} \equiv g_{i j} \widehat{\pi}^{i j} \approx 0$

for the momenta,

$\widehat{\pi}^{i j} \equiv \frac{\delta S}{\delta \dot{g}_{i j}}=\frac{2 \sqrt{g}}{\kappa^{2}} \widehat{G}^{i j k l} K_{k l}$,

in addition to the usual primary constraints, $\Phi_{\mu}$, in (10).

Then, one can find the canonical Hamiltonian, up to boundary terms,

$\widehat{H}_{C}=\int_{\Sigma_{t}} d^{D} x\left\{N \widehat{\mathcal{H}}+N_{i} \widehat{\mathcal{H}}^{i}\right\}$

with

$\widehat{\mathcal{H}} \equiv \frac{\kappa^{2}}{2 \sqrt{g}} \widehat{\pi}^{i j} \widehat{\pi}_{i j}+\sqrt{g} \mathcal{V}$,

$\widehat{\mathcal{H}}^{i} \equiv-2 \nabla_{j} \widehat{\pi}^{i j}$.

Now, the preservation of the additional primary constraint (48),

$$
\begin{aligned}
\dot{\chi} & =\left\{\chi, \widehat{H}_{C}\right\} \\
& \equiv \Psi \approx 0
\end{aligned}
$$

produces a new secondary constraint,

$$
\begin{aligned}
\Psi \equiv & \left(\frac{\kappa^{2}}{2}\right)\left(\frac{D}{2}\right) \frac{N}{\sqrt{g}} \widehat{\pi}_{m n} \widehat{\pi}^{m n} \\
& -N \sqrt{g}\left[\left(\frac{D}{2}\right) \mathcal{V}(R)-R \mathcal{V}^{\prime}(R)\right] \\
& +\sqrt{g}(D-1) \nabla^{2}\left(N \mathcal{V}^{\prime}(R)\right) \\
= & \frac{D}{2} N \widehat{\mathcal{H}}-N \sqrt{g}\left[D \mathcal{V}(R)-R \mathcal{V}^{\prime}(R)\right] \\
& +\sqrt{g}(D-1) \nabla^{2}\left(N \mathcal{V}^{\prime}(R)\right) \approx 0
\end{aligned}
$$

from (A18), in addition to the usual (reduced) Hamiltonian and momentum constraints in (12),

$\widehat{\mathcal{H}} \approx 0, \widehat{\mathcal{H}}^{i} \approx 0$.

The extended Hamiltonian is then given by

$$
\widehat{H}_{E}=\widehat{H}_{C}+\int_{\Sigma_{t}} d^{D} x\left(u_{\mu} \Phi^{\mu}+v \chi\right)
$$

with a new Lagrange multiplier $v$. The constraint algebra, (16)-(18), are reduced to

$$
\begin{aligned}
\{\widehat{\mathcal{H}}(x), \widehat{\mathcal{H}}(y)\}= & \widehat{C}^{i}(x) \nabla_{i}^{x} \delta^{D}(x-y) \\
& -\widehat{C}^{i}(y) \nabla_{i}^{y} \delta^{D}(x-y), \\
\left\{\widehat{\mathcal{H}}(x), \widehat{\mathcal{H}}_{i}(y)\right\}= & -\widehat{\mathcal{H}}(y) \nabla_{i}^{y} \delta^{D}(x-y), \\
\left\{\widehat{\mathcal{H}}_{i}(x), \widehat{\mathcal{H}}_{j}(y)\right\}= & \widehat{\mathcal{H}}_{i}(y) \nabla_{j}^{x} \delta^{D}(x-y) \\
& +\widehat{\mathcal{H}}_{j}(x) \nabla_{i}^{x} \delta^{D}(x-y),
\end{aligned}
$$

where

$$
\begin{aligned}
\widehat{C}^{i}= & \left(-\frac{\kappa^{2}}{2}\right)\left[\left(\widehat{\mathcal{H}}^{i}+2 \nabla^{i} \widehat{\pi}\right) \mathcal{V}^{\prime}(R)\right. \\
& \left.+2\left(\widehat{\pi}^{i j}-g^{i j} \widehat{\pi}\right) \nabla_{j} \mathcal{V}^{\prime}(R)\right] .
\end{aligned}
$$

Using the above reduced constraint algebra, one can find that

$$
\begin{aligned}
\dot{\hat{\mathcal{H}}} & =\left\{\widehat{\mathcal{H}}, \widehat{H}_{E}\right\} \\
& =\frac{1}{N} \nabla_{i}\left(N^{2} \widehat{C}^{i}\right)+\nabla_{i}\left(N^{i} \widehat{\mathcal{H}}\right)+\{\widehat{\mathcal{H}},\langle v \pi\rangle\}, \\
\hat{\mathcal{H}}_{i} & =\left\{\widehat{\mathcal{H}}_{i}, \widehat{H}_{E}\right\} \\
& =\widehat{\mathcal{H}} \nabla_{i} N+\nabla_{j}\left(N^{j} \widehat{\mathcal{H}}_{i}\right)+\widehat{\mathcal{H}}_{j} \nabla_{i} N^{j}+\widehat{\pi} \nabla_{i} v \approx 0,
\end{aligned}
$$

where

$$
\begin{aligned}
\{\widehat{\mathcal{H}},\langle v \pi\rangle\}= & {\left[-\frac{v}{N} \Psi+\sqrt{g}(D-1)\left(\frac{v}{N} \nabla^{2}\left(N \mathcal{V}^{\prime}(R)\right)\right.\right.} \\
& \left.\left.-\mathcal{V}^{\prime}(R) \nabla^{2} v\right)\right] .
\end{aligned}
$$

For the potential, $-\mathcal{V}(R) \equiv \Lambda+\xi R+\alpha R^{n}$, (63) becomes

$$
\begin{aligned}
\{\widehat{\mathcal{H}},\langle v \pi\rangle\}=- & {\left[\frac{v}{N} \Psi+\sqrt{g}(D-1)\left(-\frac{v}{N} \nabla^{2}(N(\xi\right.\right.} \\
& \left.\left.\left.\left.+\alpha n R^{n-1}\right)\right)+\left(\xi+\alpha n R^{n-1}\right) \nabla^{2} v\right)\right]
\end{aligned}
$$

Since we are considering the non-trivial case of $-\mathcal{V}^{\prime}(R) \equiv$ $\xi+\alpha n R^{n-1} \neq 0$, preserving the Hamiltonian constraint, $\widehat{\mathcal{H}} \approx 0$, i.e., $\dot{\hat{\mathcal{H}}} \approx 0$ in $(61)$, does not produce new constraints but determines the Lagrange multiplier $v$ : For the $\lambda$-deformed GR $(\alpha=0)$, where $\widehat{C}^{i}=0,(61)$ and (64) determine $v=N$.

On the other hand, for the preservation of the secondary constraint, $\widetilde{\Psi} \equiv \Psi_{\widehat{\mathcal{H}} \approx 0}$,

$$
\begin{aligned}
\widetilde{\Psi} \equiv & N \sqrt{g}\left[D \Lambda+(D-1) \xi R+(D-n) \alpha R^{n}\right] \\
& +\sqrt{g}(D-1) \nabla^{2}\left(N \mathcal{V}^{\prime}\right),
\end{aligned}
$$

one can find that

$$
\begin{aligned}
\dot{\widetilde{\Psi}} & =\left\{\widetilde{\Psi}, \widehat{H}_{E}\right\} \\
& =\left\{\widetilde{\Psi}, \widehat{H}_{C}\right\}+\left\{\widetilde{\Psi},\left\langle u_{t} \pi_{N}\right\rangle\right\}+\{\widetilde{\Psi},\langle v \widetilde{\pi}\rangle\},
\end{aligned}
$$

where $^{14}$

$\left\{\widetilde{\Psi},\left\langle u_{t} \pi_{N}\right\rangle\right\}=\frac{u_{t}}{N} \widetilde{\Psi}+\sqrt{g}(D-1)\left(\nabla^{2}\left(u_{t} \mathcal{V}^{\prime}\right)\right.$

\footnotetext{
${ }^{14}$ Useful relations for these computations are $\{\sqrt{g}(x), \pi(y)\}=$ $(D / 2) \sqrt{g}(x) \delta^{D}(x-y)$ and $\{R(x), \pi(y)\}=-R(x) \delta^{D}(x-y)-(D-$ 1) $\nabla_{x}^{2} \delta^{D}(x-y)$ from (A7).
} 


$$
\begin{aligned}
& \left.-\frac{u_{t}}{N} \nabla^{2}\left(N \mathcal{V}^{\prime}\right)\right) \\
\{\tilde{\Psi},\langle v \pi\rangle\}= & v N \sqrt{g}\left[\frac{D^{2}}{2} \Lambda+\frac{(D-1)(D-2)}{2} \xi R\right. \\
& \left.+\frac{(D-2)(D-2 n)}{2} \alpha R^{n}\right] \\
& +\sqrt{g}(D-1)\left[\frac{(D-2)}{2} \nabla_{i}\left(v \nabla^{i}\left(N \mathcal{V}^{\prime}\right)\right)\right. \\
& -N \nabla^{2} v((D-1) \xi \\
& \left.\left.+(D-n) \alpha n R^{n-1}\right)\right] \\
& +\alpha n(n-1)(D-1) \sqrt{g} \nabla^{2}\left[R^{n-2} N(R v\right. \\
& \left.\left.+(D-1) \nabla^{2} v\right)\right] .
\end{aligned}
$$

$$
\begin{aligned}
s & =\frac{1}{2}\left(P-2 N_{1}-N_{2}\right) \\
& =\frac{1}{2}(D+1)(D-2),
\end{aligned}
$$

which is the same as in GR. This provides the fully nonperturbative proof of the previous perturbative analysis (see also [35] for an earlier work), which does not show the extra degrees of freedom at the linear level [10]. There may exist some similarities with Case $\mathbf{A}$ due to the same physical degrees of freedom. Actually, one can consider the maximal slicing condition, i.e., $\pi=0$, for $\lambda$-deformed GR (with an arbitrary $\lambda$ ), as an example satisfying the condition, $\widetilde{C}^{i}=0$ for Case A. But, an importance difference is that Case $\mathbf{C}$ does not depend whether $\widehat{C}^{i}=0$, i.e., commuting Hamiltonian constraint $\widehat{\mathcal{H}}$, or $\widehat{C}^{i} \neq 0$, i.e., non-commuting Hamiltonian constraint $\widehat{\mathcal{H}}$ : If we consider the maximal slicing, $\pi=0$, for Case $\mathbf{B}$ with an arbitrary $\lambda \neq 1 / D$, we have basically the same results as Case $\mathbf{C}$ ! This implies that the constraint structure of Case $\mathbf{C}$ and so its number of dynamical degrees of freedom do not depend on spatially-higher-derivative terms in the potential, which are important for distinguishing Cases $\mathbf{A}$ and $\mathbf{B}$. In other words, Case $\mathbf{C}$ does not depend on the UV conformal symmetry for the Cotton square term, $C^{i j} C_{i j}$, in the Hořava's original potential $[1,2]$ and this is essentially due to the kinematic origin of the constraint, $\chi=\widehat{\pi} \approx 0$, in (48). ${ }^{15}$

$$
\begin{aligned}
\left\{\pi_{N}(x), \widehat{\mathcal{H}}(y)\right\}= & 0, \quad\left\{\pi_{N}(x), \chi(y)\right\}=0, \\
\left\{\pi_{N}(x), \widetilde{\Psi}(y)\right\}= & \widehat{\Delta}(x-y), \\
\{\widehat{\mathcal{H}}(x), \widehat{\mathcal{H}}(y)\}= & \widehat{C}^{i}(x) \nabla_{i}^{x} \delta^{D}(x-y) \\
& -\widehat{C}^{i}(y) \nabla_{i}^{y} \delta^{D}(x-y), \\
\{\widehat{\mathcal{H}}(x), \pi(y)\} \approx & \left\{\pi_{N}(x), \widetilde{\Psi}(y)\right\}, \text { etc. },
\end{aligned}
$$

whose determinant, $\operatorname{det}(\{\widehat{\chi A}, \widehat{\chi B}\}), \quad$ is generally non-vanishing,

$$
\begin{aligned}
\operatorname{det}\left\{\widehat{\chi_{A}}, \widehat{\chi_{B}}\right\} & \approx(\operatorname{det}\{\widehat{\mathcal{H}}(x), \pi(y)\})^{2}(\operatorname{det}\{\pi(x), \widehat{\mathcal{H}}(y)\})^{2} \\
& \approx(\operatorname{det}[\widehat{\Delta}(x-y) \widehat{\Delta}(y-x)])^{2},
\end{aligned}
$$

where

$$
\begin{aligned}
& \widehat{\Delta}(x-y) \\
& \equiv-\sqrt{g}\left[D \Lambda+(D-1) \xi R+(D-n) \alpha R^{n}\right] \delta^{D}(x-y) \\
& \quad+\sqrt{g}(D-1) \nabla_{y}^{2}\left[\delta^{D}(x-y)\left(\xi+\alpha n R^{n-1}\right)(y)\right] .
\end{aligned}
$$

On the other hand, the constraints, $\Gamma_{A} \equiv\left(\pi^{i}, \mathcal{H}_{i}\right) \approx 0$, are the first-class constraints, as in Cases $\mathbf{A}$ and $\mathbf{B}$. So, the resulting number of dynamical degrees of freedom is the same as in Case $\mathbf{A}$,

\section{Examples}

In this section, we consider several exact solutions for our typical potential, $-\mathcal{V}(R) \equiv \xi R+\alpha R^{n}+\Lambda$, as some explicit examples of the constraint analysis.

\subsection{Non-rotating black holes in arbitrary dimensions}

For non-rotating, spherically symmetric black holes with $N_{i} \equiv 0$, the extrinsic curvature, $K_{i j}$, and the conjugate momentum, $\pi_{i j}$, vanish so that the Hamiltonian constraint becomes simply $\mathcal{H}=\sqrt{g} \mathcal{V}(R) \approx 0$. The general solution is ' $R=$ constant', whose constant value depends on the theory parameters, $\xi, \alpha, \Lambda$, and $n$. If we consider, as an explicit example, $D=3, n=2$, i.e., $z=2$ case in $3+1$ dimensions $[36],{ }^{16}$ then the solution is given by

$d s^{2}=-N^{2}(r) d t^{2}+\frac{d r^{2}}{f(r)}+r^{2} d \theta^{2}+r^{2} \sin ^{2} \theta d \phi^{2}$

\footnotetext{
$\overline{15}$ This case corresponds to the $\dot{\beta}=0$ case in the Birkhoff's analysis of spherically symmetric system since $\pi \sim \dot{\beta}$ and the results are in a good agreement [27].

16 This can be also the solution for $z=3$ case with the Cotton square term, $C_{i j} C^{i j}$, since the Cotton tensor vanishes, $C^{i j} \equiv$ $\epsilon^{i k l} \nabla_{k}\left(R^{j}{ }_{l}-\delta^{j}{ }_{l} R / 4\right)=0$, for the spherically symmetric cases.
} 
with

$$
\begin{aligned}
N^{2} & =f=1-\frac{2 M}{r}+\left(\frac{\xi-\sqrt{\xi^{2}-4 \alpha \Lambda}}{12 \alpha}\right) r^{2}, \\
R & =\frac{-\xi+\sqrt{\xi^{2}-4 \alpha \Lambda}}{2 \alpha} .
\end{aligned}
$$

In this case, even though there are higher-derivative contributions, we have $\widetilde{C}^{i}=0$, trivially, due to $\pi_{i j}=0$ in (31). This would be a trivial example though its result is generally valid for arbitrary power $n$ and dimension $D$. If we consider the more general, spherically symmetric solutions with $N_{r} \neq 0$ (cf. [37]), it would be a more non-trivial example with the extrinsic curvature and conjugate momenta.

\subsection{Black string solutions in $3+1$ dimensions}

For stationary black strings in $3+1$ dimensions, the general ansatz is given by

$$
\begin{aligned}
d s^{2}= & \left(-N^{2}+N_{r} N^{r}+N_{\phi} N^{\phi}\right) d t^{2} \\
& +2\left(N_{r} d r+N_{\phi} d \phi\right) d t+\frac{d r^{2}}{f}+r^{2} d \phi^{2}+g d z^{2},
\end{aligned}
$$

where all the metric functions, $N, N_{i}, f$, and $g$ depend on the radial coordinate $r$. For $z=2$ black string solution which satisfies the vanishing Cotton tensor, $C_{i j}=0$, for simplicity, a simple solution with $g=$ constant,$N_{\phi}=0$, and $\lambda=1$, which is called BTZ-type black string, is given by (with $g \equiv$ 1)

$$
\begin{aligned}
& N^{2}=f=\eta r^{2}-m, \\
& N_{r}^{2}=f^{-1}\left[\delta+\frac{\kappa^{4} \mu^{2}}{64}\left(\eta^{2}-2\left(\Lambda_{W}+\omega\right)-3 \Lambda_{W}^{2}\right)\right],
\end{aligned}
$$

where $\eta, m, \delta$ are integration constants [38] and the other parameters $\kappa, \mu, \Lambda_{W}, \omega$ are introduced by the usual parametrization,

$$
\begin{aligned}
\xi & \equiv \frac{\kappa^{4} \mu^{2}\left(\Lambda_{W}+\omega\right)}{8(1-3 \lambda)}, \\
\alpha & \equiv \frac{\kappa^{2} \mu^{2}(1-4 \lambda)}{32(1-3 \lambda)}, \\
\Lambda & \equiv-\frac{2 \kappa^{2} \mu^{2} \Lambda_{W}^{2}}{8(1-3 \lambda)} .
\end{aligned}
$$

In this case, even though the extrinsic curvatures and conjugate momenta are non-vanishing, it has a constant curvature, $R \sim f^{\prime} / r=2 \eta=$ constant. Since this is the solution for $\lambda=1$, one can easily find that $\widetilde{C}^{i}=0$ in (31) is trivially satisfied ${ }^{17}$ : The first term in (31) vanishes due to $\lambda=1$ and the second term vanishes due to $R=$ constant. If we consider the more general solutions with $\lambda \neq 1$ or the $\lambda=1$

\footnotetext{
17 This result is still valid with the angular shift vector, $N_{\phi}$.
}

solution without the condition, $C_{i j}=0$, it would be more non-trivial examples with the non-constant extrinsic curvatures and conjugate momenta.

\subsection{Rotating black holes in $3+1$ dimensions}

The exact solutions for rotating black holes in $3+1$ dimensions has not been found yet. However, for slowly rotating black holes, one can consider the ansatz [39,40],

$$
\begin{aligned}
d s^{2}= & -N^{2}(r) d t^{2}+\frac{d r^{2}}{f(r)}+r^{2} d \theta^{2}+r^{2} \sin ^{2} \theta d \phi^{2} \\
& +2 a g(r) \sin ^{2} \theta d t d \phi .
\end{aligned}
$$

At the linear order in the rotation parameter $a$, the solution of $g(r)$ for $n=2$ is given by

$g(r)=\sigma r^{2}+\frac{\gamma}{r}$

with the integration parameters, $\sigma, \gamma$, and the same solution of $N^{2}=f(r)$ as in (74). In this case, the extrinsic curvature has a non-vanishing component, $K_{r \phi}=\mathcal{O}(a)$ but $K=0, \pi=0$, at the linear order in ' $a$ '. Since the curvature scalar is constant as in (75), we will have the case $\widetilde{C}^{i}=0$ again and this result is valid for arbitrary power $n$ and dimensions $D .{ }^{18}$ If we consider higher orders in the rotation parameter $a$ so that $K$ or $\pi$ is non-vanishing or $R$ is non-constant, it would be a more non-trivial example.

\subsection{Rotating black holes in $2+1$ dimensions}

For stationary black holes in $2+1$ dimensions, the most general ansatz is given by

$$
\begin{aligned}
d s^{2}= & \left(-N^{2}+N_{r} N^{r}+N_{\phi} N^{\phi}\right) d t^{2}+2\left(N_{r} d r+N_{\phi} d \phi\right) d t \\
& +\frac{d r^{2}}{f}+r^{2} d \phi^{2}
\end{aligned}
$$

where all the metric functions, $N, N_{i}, f$, depend on the radial coordinate $r$, as in (76), due to the symmetry property in $2+1$ dimensions. The general solution for the metric (82) is not known yet. For the simple case, however, of $N_{r}=0$, one can find that the there is non-vanishing conjugate momentum, $\pi^{\phi r}=(f / 2 N)\left(N^{\phi}\right)^{\prime}$, whose trace is vanishing trivially, $\pi=$ 0 , with the solution (for the $n=2$ case) $[41,42]$

\footnotetext{
$\overline{18}$ In $[39,40], \lambda=1, \Lambda_{W}=0$ case for the IR-modified Hořava gravity was considered. But our result is generally valid for arbitrary $\lambda$ and $\Lambda_{W}$.
} 


$$
\begin{aligned}
f= & -\mathcal{M}+\frac{b r^{2}}{2}\left[1-\sqrt{a+\frac{c}{r^{4}}}+\sqrt{\frac{c}{r^{4}}} \ln \left(\sqrt{\frac{c}{a r^{4}}}\right.\right. \\
& \left.\left.+\sqrt{1+\frac{c}{a r^{4}}}\right)\right], \\
N^{2} / f= & 1 /\left(1+\frac{c}{a r^{4}}\right), \\
N^{\phi}= & -\frac{\mathcal{J}}{2} \sqrt{\frac{a}{c}} \ln \left[\sqrt{\frac{c}{a r^{4}}}+\sqrt{1+\frac{c}{a r^{4}}}\right],
\end{aligned}
$$

where

$a=1-\frac{4 \alpha \Lambda}{\xi^{2}}, b=\frac{\xi}{2 \alpha}, c=\frac{2 \alpha \mathcal{J}^{2}}{\xi^{2}}$.

In this case, even though $K=0, \pi=0$ trivially, the curvature scalar is non-constant,

$R=-\frac{f^{\prime}}{r}=-b\left(1-\sqrt{a+\frac{c}{r^{4}}}\right)$,

so that we have a non-vanishing component of $\widetilde{C}^{i}$,

$\widetilde{C}^{\phi} \sim \alpha \pi^{\phi r} \partial_{r} R \neq 0$.

But, it easy to find that the constraint $\widetilde{\Omega} \approx 0$ is satisfied again

$\widetilde{\Omega} \sim \partial_{\phi}\left(N^{2} \widetilde{C}^{\phi}\right)=0$,

due to the spherical symmetry, i.e., no $\phi$-dependence, in the solution. It is important to note that the non-vanishing $\widetilde{C}^{i}$ is the genuine result of higher-derivative terms $(\alpha \neq 0)$ with a rotation $\left(\pi^{\phi r} \neq 0\right)$. In other words, if we turn off the higherderivative term, i.e., $\alpha=0$, similarly to BTZ black hole in $\mathrm{GR}$, the system is reduced to the case with the vanishing $\widetilde{C}^{i} \sim \xi \widehat{\lambda} \nabla^{i} \pi=0$. Of course, this does not correspond to Case $\mathbf{B}$ but Case $\mathbf{C}$, due to the fact of $\pi=0$ for the solution (83), as can be seen by checking the constraint, $\widetilde{\Psi} \approx 0$, in (65). However, if we consider the $N_{r} \neq 0$ case, one obtains $\pi \neq 0$ generally so that it may correspond to a "genuine" case of Case B. This example would show the importance of higher-derivative terms for Case $\mathbf{B}$, where the extra scalar graviton mode is involved.

\section{Concluding remarks}

We study the full constraint analysis of the non-projectable Hořava gravity whose potential, $\mathcal{V}(R)$, is an arbitrary function of the (intrinsic) Ricci scalar $R$ in arbitrary dimensions but without the extension terms which depend on the proper acceleration $a_{i}$, for simplicity. We find that there are generally three distinct cases in this theory, depending on

(i) whether the Hamiltonian constraint, $\mathcal{H} \approx 0$, generates new (second-class) constraints (Cases A, C) or just fixes the associated Lagrange multipliers (Case $\mathbf{B}$ ), or (ii) whether the IR Lorentz-deformation parameter $\lambda$ is at the conformal point, $\lambda=1 / D$ (Case $\mathbf{C}$ ), or not (Cases A, B).

We find that, for Cases $\mathbf{A}$ and $\mathbf{C}$, the dynamical degrees of freedom of Hořava gravity are the same as in GR, while for Case $\mathbf{B}$, there is one extra phase-space degree of freedom, representing an extra (odd) scalar graviton mode. This would achieve the dynamical consistency of a restricted model at the fully non-linear level and be positive in resolving the long-standing debates about the extra graviton modes of the Hořava gravity. Several further remarks about other challenging problems are in order.

1. We have obtained the new "extended" constraint algebra for the Hamiltonian and momentum constraints, (16)-(18) ((57)-(59) for Case C) or (19)-(21), for the non-projectable Hořava gravity whose potential is an arbitrary function of the Ricci scalar $R$. The structure of the newly obtained "extended" constraints algebra seems to be generic to Hořava gravity itself, analogous to that of general higher-curvature gravities [25,26], where $C^{i}$ becomes the momentum constraint $\mathcal{H}^{i}$ with higher-curvature corrections. An important difference is that the momentum constraint $\mathcal{H}^{i}$ in Horrava is the same form as in GR with no higher-derivative corrections and satisfies the same algebra as in GR [23] but the full algebra with the Hamiltonian constraint, $\mathcal{H}$, is not closed, whereas the momentum constraints, $\mathcal{H}^{i} \equiv C^{i}$, in generic higher-curvature gravities satisfy exactly the same closed algebra as in GR, "as has been argued generically in [43]", even with the generic higher-curvature terms which include Riemann tensors also $[25,26,44]$. We suspect that a similar general argument in Hořava gravity exists also so that the extended constraint algebra reflects the generic spacetime structure of our Lorentz violating gravities with Ricci and Riemann tensors, $R_{i j}, R_{i j k l}$, etc., i.e., with the potential, $\mathcal{V}\left(R, R_{i j}, R_{i j k l}, \ldots\right),{ }^{19}$ and its general proof would be a challenging problem.

2. Our constraint analysis shows the dynamical degrees of freedom in a restricted model of the Hořava gravity at the fully non-linear level. Comparison to the previous linear perturbation analyses $[8,10,33]$, which do not show the extra degrees of freedom, implies that the extra modes would be the genuine consequence of non-linear effect (around the homogenous background) with Lorentz-violating higher-derivative terms [27]: For a direct proof in the $n=2$ constraint algebra, see [15]. On the other hand, it has been also argued that the extra mode may appear when considering (even linear) perturbations around the spatially-inhomogeneous and time-dependent background [5], even with the IR Lorentzviolating terms only, as anticipated from the usual connection

\footnotetext{
19 This implies the generic absence of the third and second-derivative terms in (2.24) of [4], which is the case of $\lambda=1$ and $\mathcal{V} \sim C_{i j} C^{i j}$.
} 
between non-linear perturbations for a homogenous background and linear perturbations for a corresponding inhomogeneous background. ${ }^{20}$ This may be in contrast to the Birkhoff's theorem analysis in spherically symmetric, vacuum configuration which does not show time-dependent solutions, representing the "extra gravitational" modes [27]. However, this might be due to its high symmetry, i.e., lower inhomogeneity, and there might exist still some possibilities for higher inhomogeneities: This might be consistent with a related analysis in [15] which does not show the extra mode only up to the first order of inhomogeneity, $L^{-1}$. So, a direct proof of the argument of [5] in the generic constraint algebra would be an interesting open problem. The role of non-linear, UV scalar graviton in Big Bang cosmology and gravitational wave physics for compact objects with strong gravities, like black holes and neutron stars, would be also a challenging problem.

3. In the literature, there have been claims of inconsistencies of the Hořava gravity, in its original form. It seems that some originate from the limited analysis, like linear approximations [7], or incomplete constraint analysis [4,11]. Here, we consider the second case, ${ }^{21}$ especially about the claim in [11], which seems to be the most rigorous criticism against the (non-projectable) Hořava gravity. The basic claim in [11] is that " $N=0$ is the only possible solution" for the constraint (4.1), $\nabla_{i}\left(N^{2} \nabla^{i} \pi\right) \approx 0$, which corresponds to our constraint (30), $\widetilde{\Omega} \equiv \nabla_{i}\left(N^{2} \widetilde{C}^{i}\right) \approx 0$, for the asymptotically flat $(\Lambda=0)$ and $\lambda$-deformed GR, otherwise $N$ blows up at infinity, generically. ${ }^{22}$ But, the elaborate analysis in [11] is just another proof of an adequacy of the condition, $\pi=0$, in that example, which is the only remaining solution for the constraint (4.1) in [11] or (30) in this paper, as has been argued also in $[14,15]$. Actually, contrary to the argument in [11], the perturbations from $\pi=0$ are not arbitrary but restricted by another constraint (33), $\widetilde{\Xi} \approx 0$ in this paper (or (20) in [14]), when $\lambda \neq 1$. Moreover, when the metric is not asymptotically flat $(\Lambda \neq 0)$, the argument of [14] (and possibly of [11] or [5] also) would not be valid generally, as can be seen in the general, $(2+1)$-dimensional solution, (82) with $N_{r} \neq 0$, which shows the non-vanishing $\pi$ and $N$.

4. With the full set of constraints, we can now consider Feynman's path-integral for the S-matrix elements [45,46], whose Hamiltonian expression for Case $\mathbf{A}$, for example, is given by

\footnotetext{
${ }^{20}$ MIP thank Koyama and Gumrukcuoglu for discussion about this matter.

21 The first case has been discussed in several places, like [15] (footnote $3)$ and [4] (Note added).

22 This result corresponds to exactly what has been argued in [5], which shows "instabilities for perturbations around a non-vanishing $K$ background", though given at the linear level.
}

$$
\begin{aligned}
Z= & \int \mathcal{D} g_{i j} \mathcal{D} \pi^{i j} \mathcal{D} N \mathcal{D} N_{i} \delta\left(\mathcal{H}_{i}\right) \delta\left(\gamma_{j}\right) \delta(\mathcal{H}) \delta(\widetilde{\Omega}) \\
& \times \int \mathcal{D} c_{j} \mathcal{D} \bar{c}_{i} \exp \left\{i \int d t d^{D} x \bar{c}_{i}\left[\left.\left\{\mathcal{H}_{i}, \gamma_{j}\right\}\right|_{\pi^{i} \approx 0}\right] c_{j}\right\} \\
& \times \int \mathcal{D} b \mathcal{D} \bar{b} \exp \left\{i \int d t d^{D} \times \bar{b}\left[\left.\{\mathcal{H}, \widetilde{\Omega}\}\{\widetilde{\Omega}, \mathcal{H}\}\right|_{\pi_{N} \approx 0}\right] b\right. \\
& +i S / \hbar\},
\end{aligned}
$$

where we have integrated out for the primary constraints $\pi_{N}, \pi^{i} \approx 0$ with the Faddeev-Popov's anti-commuting fields, $c_{j}, \bar{c}_{i}, b, \bar{b}$, the gauge-fixing conditions, $\gamma_{j}=0$, for the first-class constraint, $\mathcal{H}_{i} \approx 0$, and the determinant factor for the second-class constraints, $\left(\operatorname{det}\left\{\chi_{A}, \chi_{B}\right\}\right)^{1 / 2} \approx$ $\operatorname{det}(\{\mathcal{H}, \widetilde{\Omega}\}\{\widetilde{\Omega}, \mathcal{H}\})$. One can obtain similarly the path integral for Cases $\mathbf{B}$ and $\mathbf{C}$ also. It would be desirable to study the renormalizability for the non-projectable Hořava gravity, based on the above S-matrix elements, beyond the recent proof of (perturbative) renormalizability for the projectable cases [47-49]. ${ }^{23}$

5. In the canonical quantization with the second-class constraints, we need to compute the Dirac brackets [17], ${ }^{24}$ whose definition for Case $\mathbf{A}$, for example, is given by, for any variable $P, Q$,

$$
\begin{aligned}
& \{P, Q\}^{*} \equiv\{P, Q\} \\
& \quad-\int d^{D} z \int d^{D} w\left\{P, \chi_{A}(z)\right\} C_{A B}^{-1}(z, w)\left\{\chi_{B}(w), Q\right\},
\end{aligned}
$$

where $C_{A B}^{-1}$ is defined as $\int d^{D} z C_{A B}^{-1}(x, z) C_{B C}(z, y)=$ $\int d^{D} z C_{C B}(y, z) C_{B A}^{-1}(z, x)=\delta_{A C} \delta^{D}(x-y)$ for the Poisson brackets of the second-class constraints $C_{A B} \equiv\left\{\chi_{A}, \chi_{B}\right\}$, and given by

$C_{A B}^{-1}(x, z)=\left(\begin{array}{cccc}0 & -\Delta^{-1}(z-x) & 0 & 0 \\ \Delta^{-1}(x-z) & 0 & 0 & 0 \\ 0 & 0 & 0 & -\Delta^{-1}(z-x) \\ 0 & 0 & \Delta^{-1}(x-z) & 0\end{array}\right)$

$\Delta^{-1}$, defined by $\int d^{D} z \Delta^{-1}(x-z) \Delta(z-y)=\int d^{D} z \Delta(y-$ $z) \Delta^{-1}(z-x)=\delta^{D}(x-y)$. The bracket satisfies $\left\{\chi_{A}, Q\right\}^{*}=$ 0 for any variable $Q$ so that the second-class constraints, $\chi_{A} \approx 0$, can be imposed consistently in the Hamiltonian dynamics, i.e., $\{\mathcal{H}, \mathcal{H}\}^{*}=0,\left\{\mathcal{H}, \mathcal{H}_{i}\right\}^{*}=0$, etc., ${ }^{25}$ which corresponds to the delta-function insertions for the constraints, $\chi_{A} \approx 0$, in the path integral, (88). If we consider the gauge-fixing conditions as in the path integral formalism, we

\footnotetext{
${ }^{23}$ For an earlier work on non-projectable case in $(2+1)$-dimensions, see also [50] (Case C).

24 After this paper appeared in the archive, we became aware of [52] in which the Dirac brackets were computed for the linearized Hořava gravity. We thank Ghosh for informing us about his work.

25 This algebra looks like that of the "ultra-local" theory of gravity $[1,2,51]$.
} 
can obtain further corrections to the Dirac brackets. Then the Dirac's quantization rule is given by $[\widehat{P}, \widehat{Q}] \equiv(i / \hbar)\{P, Q\}^{*}$ for the quantum operators, $\widehat{P}$ and $\widehat{Q}$, corresponding to classical variables $P$ and $Q$, respectively, with the "appropriate" operator orderings. One can consider also Cases $\mathbf{B}$ and $\mathbf{C}$ similarly, but it would be more involved for the former case.

Note added: After finishing this paper, a related paper [53] appeared which is overlapping with ours for $D=2$ case. But due to the (full) $a_{i}$ extensions in [53], it shows a different constraint structure, as noted in our footnote No. 9.

Acknowledgements This work was supported by Basic Science Research Program through the National Research Foundation of Korea (NRF) funded by the Ministry of Education, Science and Technology (2016R1A2B401304, 2020R1A2C1010372, 2020R1A6A1A03047877). DOD was also supported by the National Natural Science Foundation of China under Grant No. 11875136 and the Major Program of the National Natural Science Foundation of China under Grant No. 11690021.

Data Availability Statement This manuscript has no associated data or the data will not be deposited. [Authors' comment: There is no data for deposition.]

Open Access This article is licensed under a Creative Commons Attribution 4.0 International License, which permits use, sharing, adaptation, distribution and reproduction in any medium or format, as long as you give appropriate credit to the original author(s) and the source, provide a link to the Creative Commons licence, and indicate if changes were made. The images or other third party material in this article are included in the article's Creative Commons licence, unless indicated otherwise in a credit line to the material. If material is not included in the article's Creative Commons licence and your intended use is not permitted by statutory regulation or exceeds the permitted use, you will need to obtain permission directly from the copyright holder. To view a copy of this licence, visit http://creativecomm ons.org/licenses/by/4.0/.

Funded by SCOAP 3 .

\section{Appendix A: Computing $\{\mathcal{H}, \mathcal{H}\},\left\{\mathcal{H}, \mathcal{H}_{i}\right\}$, and $\left\{\mathcal{H}_{i}, \mathcal{H}_{j}\right\}$}

In this Appendix, we compute the constraint algebra, $\{\mathcal{H}, \mathcal{H}\},\left\{\mathcal{H}, \mathcal{H}_{i}\right\}$, and $\left\{\mathcal{H}_{i}, \mathcal{H}_{j}\right\}$ in (16)-(18). To this ends, it is useful to consider the variations of the smeared constraints, $\langle\eta \mathcal{H}\rangle \equiv \int d^{D} x \eta \mathcal{H} \approx 0$, etc., with the smearing functions, $\eta, \eta_{i}$ (neglecting the boundary terms),

$$
\begin{aligned}
\delta\langle\eta \mathcal{H}\rangle & =\left\langle A^{k l} \delta g_{k l}+B_{k l} \delta \pi^{k l}\right\rangle, \\
\delta\left\langle\eta^{i} \mathcal{H}_{i}\right\rangle & =\left\langle C^{k l} \delta g_{k l}+D_{k l} \delta \pi^{k l}\right\rangle .
\end{aligned}
$$

After long computations, one can obtain the coefficients, $A, B, C, D$ as follows:

$$
\begin{aligned}
A^{k l} \equiv & \frac{\delta\langle\eta \mathcal{H}\rangle}{\delta g_{k l}}=\left(\frac{\kappa^{2}}{2}\right) \frac{\eta}{\sqrt{g}}\left[-\frac{1}{2} g^{k l}\left(\pi_{m n} \pi^{m n}-\tilde{\lambda} \pi^{2}\right)\right. \\
& \left.+2\left(\pi^{k m} \pi_{m}^{l}-\tilde{\lambda} \pi \pi^{k l}\right)\right] \\
& +\eta \sqrt{g}\left[\frac{1}{2} g^{k l} \mathcal{V}(R)-R^{k l} \mathcal{V}^{\prime}(R)\right]
\end{aligned}
$$

$$
\begin{aligned}
& +\sqrt{g}\left[\nabla^{k} \nabla^{l}\left(\eta \mathcal{V}^{\prime}(R)\right)-g^{k l} \nabla_{m} \nabla^{m}\left(\eta \mathcal{V}^{\prime}(R)\right)\right] \\
B_{k l} \equiv & \frac{\delta\langle\eta \mathcal{H}\rangle}{\delta \pi^{k l}}=\left(\frac{\kappa^{2}}{2}\right) \frac{2 \eta}{\sqrt{g}}\left(\pi_{k l}-\tilde{\lambda} g_{k l} \pi\right) \\
C^{k l} \equiv & \frac{\delta\left\langle\eta^{i} \mathcal{H}_{i}\right\rangle}{\delta g_{k l}}=-\nabla_{m}\left(\eta^{m} \pi^{k l}\right) \\
& +\left(\nabla_{m} \eta^{k}\right) \pi^{l m}+\left(\nabla_{m} \eta^{l}\right) \pi^{k m} \\
D_{k l} \equiv & \frac{\delta\left\langle\eta^{i} \mathcal{H}_{i}\right\rangle}{\delta \pi^{k l}}=\nabla_{k} \eta_{l}+\nabla_{l} \eta_{k} .
\end{aligned}
$$

As a byproduct, one can also obtain a useful formula,

$$
\begin{aligned}
\left\{R(x), \pi^{k l}(y)\right\}= & \frac{\delta R(x)}{\delta g_{k l}(y)}=-R^{k l}(x) \delta^{D}(x-y) \\
& +\nabla_{x}^{k} \nabla_{x}^{l} \delta^{D}(x-y)-g^{k l} \nabla_{x}^{2} \delta^{D}(x-y) .
\end{aligned}
$$

Then, after some manipulations, one can find the Poisson bracket algebras for the smeared constraints as follows:

$$
\begin{aligned}
& \{\langle\eta \mathcal{H}\rangle,\langle\zeta \mathcal{H}\rangle\} \\
& \quad=\int d^{D} z\left[\frac{\delta\langle\eta \mathcal{H}\rangle}{\delta g_{k l}(z)} \frac{\delta\langle\zeta \mathcal{H}\rangle}{\delta \pi^{k l}(z)}-\frac{\delta\langle\eta \mathcal{H}\rangle}{\delta \pi^{k l}(z)} \frac{\delta\langle\zeta \mathcal{H}\rangle}{\delta g_{k l}(z)}\right] \\
& =\left\langle\left(\eta \nabla_{i} \zeta-\zeta \nabla_{i} \eta\right) C^{i}\right\rangle, \\
& \left\{\langle\eta \mathcal{H}\rangle,\left\langle\zeta^{i} \mathcal{H}_{i}\right\rangle\right\} \\
& =\int d^{D} z\left[\frac{\delta\langle\eta \mathcal{H}\rangle}{\delta g_{k l}(z)} \frac{\delta\left\langle\zeta^{i} \mathcal{H}_{i}\right\rangle}{\delta \pi^{k l}(z)}-\frac{\delta\langle\eta \mathcal{H}\rangle}{\delta \pi^{k l}(z)} \frac{\delta\left\langle\zeta^{i} \mathcal{H}_{i}\right\rangle}{\delta g_{k l}(z)}\right] \\
& =-\left\langle\zeta^{i} \nabla_{i} \eta \mathcal{H}\right\rangle, \\
& \left\{\left\langle\zeta^{i} \mathcal{H}_{i}\right\rangle,\left\langle\zeta^{j} \mathcal{H}_{j}\right\rangle\right\} \\
& =\int d^{D} z\left[\frac{\delta\left\langle\zeta^{i} \mathcal{H}_{i}\right\rangle}{\delta g_{k l}(z)} \frac{\delta\left\langle\zeta^{j} \mathcal{H}_{j}\right\rangle}{\delta \pi^{k l}(z)}-\frac{\delta\left\langle\zeta^{i} \mathcal{H}_{i}\right\rangle}{\delta \pi^{k l}(z)} \frac{\delta\left\langle\zeta^{j} \mathcal{H}_{j}\right\rangle}{\delta g_{k l}(z)}\right] \\
& =\left\langle\left(\zeta^{i} \nabla_{i} \zeta^{j}-\zeta^{i} \nabla_{i} \zeta^{j}\right) \mathcal{H}_{j}\right\rangle,
\end{aligned}
$$

where

$$
\begin{aligned}
C^{i}= & \left(-\frac{\kappa^{2}}{2}\right)\left[\left(\mathcal{H}^{i}+2 \widehat{\lambda} \nabla^{i} \pi\right) \mathcal{V}^{\prime}(R)\right. \\
& \left.+2\left(\pi^{i j}-\widehat{\lambda} g^{i j} \pi\right) \nabla_{j} \mathcal{V}^{\prime}(R)\right] .
\end{aligned}
$$

Now, one can easily check that the Poisson algebra for the local constraints are given by (16)-(18):

$$
\begin{aligned}
\{\mathcal{H}(x), \mathcal{H}(y)\}= & C^{i}(x) \nabla_{i}^{x} \delta^{D}(x-y) \\
& -C^{i}(y) \nabla_{i}^{y} \delta^{D}(x-y), \\
\left\{\mathcal{H}(x), \mathcal{H}_{i}(y)\right\}= & -\mathcal{H}(y) \nabla_{i}^{y} \delta^{D}(x-y), \\
\left\{\mathcal{H}_{i}(x), \mathcal{H}_{j}(y)\right\}= & \mathcal{H}_{i}(y) \nabla_{j}^{x} \delta^{D}(x-y) \\
& +\mathcal{H}_{j}(x) \nabla_{i}^{x} \delta^{D}(x-y) .
\end{aligned}
$$

Moreover, from (A1)-(A6), one can easily obtain the dynamical equations of motion (13)-(14) as follows, 


$$
\begin{aligned}
\dot{g}_{i j}= & \left\{g_{i j},\left\langle N \mathcal{H}+N_{i} \mathcal{H}^{i}\right\rangle\right\}=\frac{\delta\langle N \mathcal{H}\rangle}{\delta \pi^{i j}}+\frac{\delta\left\langle N_{i} \mathcal{H}^{i}\right\rangle}{\delta \pi^{i j}} \\
= & \left(\frac{\kappa^{2}}{2}\right) \frac{2 N}{\sqrt{g}}\left(\pi_{i j}-\tilde{\lambda} g_{i j} \pi\right)+\nabla_{i} N_{j}+\nabla_{j} N_{i}, \quad(\mathrm{~A} 15) \\
\dot{\pi}^{i j}= & \left\{\pi^{i j},\left\langle N \mathcal{H}+N_{i} \mathcal{H}^{i}\right\rangle\right\}=-\frac{\delta\langle N \mathcal{H}\rangle}{\delta g_{i j}}-\frac{\delta\left\langle N_{i} \mathcal{H}^{i}\right\rangle}{\delta g_{i j}} \\
= & \left(\frac{\kappa^{2}}{2}\right) \frac{N}{\sqrt{g}}\left[\frac{1}{2} g^{i j}\left(\pi_{m n} \pi^{m n}-\tilde{\lambda} \pi^{2}\right)\right. \\
& \left.-2\left(\pi^{i m} \pi_{m}^{j}-\tilde{\lambda} \pi \pi^{i j}\right)\right] \\
& -N \sqrt{g}\left[\frac{1}{2} g^{i j} \mathcal{V}(R)-R^{i j} \mathcal{V}^{\prime}(R)\right] \\
& -\sqrt{g}\left[\nabla^{i} \nabla^{j}\left(N \mathcal{V}^{\prime}(R)\right)-g^{i j} \nabla_{m} \nabla^{m}\left(N \mathcal{V}^{\prime}(R)\right)\right] \\
& +\nabla_{m}\left(N^{m} \pi^{i j}\right)-\left(\nabla_{m} N^{i}\right) \pi^{j m}-\left(\nabla_{m} N^{j}\right) \pi^{i m} .
\end{aligned}
$$

Combining (A15) and (A16), one can also obtain the dynamical equation for $\sqrt{g}$ and the momentum trace $\pi$ as follows,

$$
\begin{aligned}
\sqrt{\dot{g}}= & \left(\frac{\kappa^{2}}{2}\right) \frac{N \pi}{1-D \lambda}+2 \nabla_{i} N^{i} \\
\dot{\pi}= & \left(\frac{\kappa^{2}}{2}\right)\left(\frac{D}{2}\right) \frac{N}{\sqrt{g}}\left(\pi_{m n} \pi^{m n}-\tilde{\lambda} \pi^{2}\right)+\nabla_{m}\left(N^{m} \pi\right) \\
& -N \sqrt{g}\left[\left(\frac{D}{2}\right) \mathcal{V}(R)-R \mathcal{V}^{\prime}(R)\right] \\
& +\sqrt{g}(D-1) \nabla^{2}\left(N \mathcal{V}^{\prime}(R)\right) \\
= & \frac{D}{2} N \mathcal{H}-N \sqrt{g}\left[D \mathcal{V}(R)-R \mathcal{V}^{\prime}(R)\right] \\
& +\sqrt{g}(D-1) \nabla^{2}\left(N \mathcal{V}^{\prime}(R)\right)+\nabla_{m}\left(N^{m} \pi\right)
\end{aligned}
$$

\section{Appendix B: More details of computations in Sect. 3 A}

In this Appendix, we present some more details of the computations in Sect. 3 A.

First, we consider the variations of the smeared constraints, $\langle\eta \widetilde{\Omega}\rangle \equiv \int d^{D} x \eta \widetilde{\Omega}$ and $\langle\zeta \widetilde{\Xi}\rangle \equiv \int d^{D} x \zeta \widetilde{\Xi}$ with the smearing functions $\eta$ and $\zeta$,

$\delta\langle\eta \widetilde{\Omega}\rangle=\left\langle E^{m n} \delta g_{m n}+F_{m n} \delta \pi^{m n}\right\rangle$,

$\delta\langle\zeta \widetilde{\Xi}\rangle=\left\langle G^{m n} \delta g_{m n}+H_{m n} \delta \pi^{m n}\right\rangle$.

After quite tedious computations, we obtain the coefficients, $E, F, G, H$ as follows ${ }^{26}$ :

$E^{m n} \equiv \frac{\delta\langle\eta \widetilde{\Omega}\rangle}{\delta g_{m n}}=-\left(\frac{\kappa^{2}}{2}\right) 2 \widehat{\lambda} \mathcal{V}^{\prime}(R)\left[\pi^{m n} \nabla_{i}\left(N^{2} \nabla^{i} \eta\right)\right.$

\footnotetext{
${ }^{26}$ Due to the messy expressions, we have used " $x A c t$ " for crosschecking our computations.
}

$$
\begin{aligned}
& \left.-\frac{1}{2} g^{m n} \nabla_{i}\left(N^{2} \pi \nabla^{i} \eta\right)+N^{2} \nabla^{(m} \pi \nabla^{n)} \eta\right] \\
& -\left(\frac{\kappa^{2}}{2}\right) 2 \widehat{\lambda}\left\langle\eta \nabla _ { i } \left[ N^{2} \nabla^{i} \pi \frac{\delta \mathcal{V}^{\prime}(R)}{\delta g_{m n}}\right.\right. \\
& +N^{2}\left(\pi^{i j}-\widehat{\lambda} g^{i j} \pi\right) \frac{\delta \nabla_{j} \mathcal{V}^{\prime}(R)}{\delta g_{m n}} \\
& \left.\left.-N^{2}\left(\delta^{i j}{ }_{m n} \pi+g^{i j} \pi^{m n}\right) \nabla_{j} \mathcal{V}^{\prime}(R)\right]\right\rangle, \\
& F_{m n} \equiv \frac{\delta\langle\eta \widetilde{\Omega}\rangle}{\delta \pi^{m n}}=-\left(\frac{\kappa^{2}}{2}\right) 2 \widehat{\lambda} \mathcal{V}^{\prime}(R) \nabla_{i}\left[g_{m n} N^{2} \nabla^{i} \eta\right] \\
& -\left(\frac{\kappa^{2}}{2}\right) 2\left\langle\eta \nabla_{i}\left[N^{2}\left(\delta^{i j}{ }_{m n}-\widehat{\lambda} g^{i j} g_{m n}\right) \nabla_{j} \mathcal{V}^{\prime}(R)\right]\right\rangle \text {, } \\
& G^{m n} \equiv \frac{\delta\langle\zeta \widetilde{\Xi}\rangle}{\delta g_{m n}}=\left(\frac{\kappa^{2}}{2}\right) 2(D-1) \xi^{2} \widehat{\lambda} \sqrt{g}\left\{\frac{1}{2} g^{m n} N^{2}\right. \\
& \times \nabla_{i} \zeta\left(\nabla^{i} \nabla^{j} \nabla_{j} N-N \nabla^{i} R-R \nabla^{i} N\right) \\
& +N^{2}\left[\nabla_{i}\left(N R^{m n}\right) \nabla^{i} \zeta-\nabla^{(m} \nabla_{i} \nabla^{i} N \nabla^{n)} \zeta\right. \\
& \left.+\nabla^{(m}(N R) \nabla^{n)} \zeta-\nabla_{i} \nabla^{m} \nabla^{n} N \nabla^{i} \zeta\right] \\
& +\frac{\Lambda D}{2 \xi(D-1)}\left[2 N^{2} \nabla^{(m} N \nabla^{n)} \zeta-g^{m n} N^{2} \nabla^{i} N \nabla_{i} \zeta\right] \\
& +\nabla_{i}\left[N ^ { 2 } \left(X^{i m n k l} \nabla_{j} \nabla_{k} N \nabla^{j} \zeta\right.\right. \\
& \left.\left.+2 X^{i m n k j l} \nabla_{l} \nabla_{k} N \nabla_{j} \zeta-N R^{m n} \nabla^{i} \zeta\right)\right] \\
& +\nabla_{k} \nabla_{i}\left[N^{2}\left(X^{k m n l} l_{l}^{i}-X^{k m n i l}{ }_{l}\right) \nabla_{j} N \nabla^{j} \zeta\right. \\
& \left.-N^{2} X^{k m n j l}{ }_{l} \nabla_{j} N \nabla^{i} \zeta\right] \\
& \left.+\nabla_{k} \nabla_{j} \nabla_{i}\left[N^{3} \nabla^{i} \zeta\left(X^{k m n j l} l-X^{k m n l} l^{j}\right)\right]\right\} \\
& +\left(\frac{\kappa^{2}}{2}\right)^{2} \frac{\xi \widehat{\lambda}}{(\lambda D-1) \sqrt{g}}\left\{( 2 \lambda + 1 ) \left[2 \pi^{m n} \pi\right.\right. \\
& \times \nabla_{i}\left(N^{3} \nabla^{i} \zeta\right)-g^{m n} \nabla_{i}\left(N^{3} \pi^{2} \nabla^{i} \zeta\right) \\
& \left.+g^{m n} N^{3} \pi \nabla^{i} \pi \nabla_{i} \zeta+2 N^{3} \pi \nabla^{(m} \pi \nabla^{n)} \zeta\right] \\
& +N^{2}\left[g^{m n} \pi^{2} \nabla_{i} N \nabla^{i} \zeta+2 \pi^{2} \nabla^{(m} N \nabla^{n)} \zeta\right. \\
& \left.-4 \pi^{m n} \pi \nabla_{i} N \nabla^{i} \zeta\right]+4(\lambda D-1) \\
& \left.\times\left[\left(\frac{1}{2} g^{m n} \pi^{i j} \pi-\pi^{m n} \pi^{i j}\right) \nabla_{j}\left(N^{3} \nabla_{i} \zeta\right)\right]\right\} \\
& + \text { (higher-derivative contributions), } \\
& H_{m n} \equiv \frac{\delta\langle\zeta \widetilde{\Xi}\rangle}{\delta \pi^{m n}}=\left(\frac{\kappa^{2}}{2}\right)^{2} \frac{4 \xi}{\sqrt{g}} \widehat{\lambda} \\
& \left\{N^{3} \nabla_{(m} \pi \nabla_{n)} \zeta-g_{m n} \nabla_{i}\left(N^{3} \pi^{i j} \nabla_{j} \zeta\right)\right. \\
& +\frac{N^{2} g_{m n}}{2(\lambda D-1)}\left[(2 \lambda+1) N \pi \nabla_{i} \nabla^{i} \zeta\right. \\
& \left.\left.+(6 \lambda+1) \pi \nabla_{i} N \nabla^{i} \zeta\right]\right\}
\end{aligned}
$$


+(higher-derivative contributions),

(B6)

where $f_{(i j)} \equiv\left(f_{i j}+f_{j i}\right) / 2,-\mathcal{V}^{\prime}(R) \equiv\left(\xi+\alpha n R^{n-1}\right)$, and

$$
\begin{aligned}
-\frac{\delta \mathcal{V}^{\prime}(R)(y)}{\delta g_{m n}(x)}= & \alpha n(n-1) R^{n-2} \frac{\delta R(y)}{\delta g_{m n}(x)} \\
= & -\alpha n(n-1) R^{n-2}\left(R^{m n} \delta^{D}(x-y)\right. \\
& \left.+A^{m n p q}{ }_{k l} g^{k l} \nabla_{p} \nabla_{q} \delta^{D}(x-y)\right) \\
= & -\alpha n(n-1) R^{n-2}\left[R^{m n} \delta^{D}(x-y)\right. \\
& \left.-\left(\nabla^{n} \nabla^{m}-g^{m n} \nabla^{2}\right) \delta^{D}(x-y)\right], \\
A^{m n p q}{ }_{k l} \equiv & \left.g^{p i} X^{q m n}{ }_{i k l}-g^{i j} X^{q m n}{ }_{i j(k} \delta^{p} l\right), \\
X^{m i j}{ }_{n k l} \equiv & \frac{1}{2}\left[g^{m}{ }_{k} g^{(i}{ }_{l} g^{j)}{ }_{n}+g^{m}{ }_{l} g^{(i}{ }_{n} g^{j)}{ }_{k}\right. \\
& \left.-g^{m}{ }_{n} g^{(i}{ }_{k} g^{j)}{ }_{l}\right] .
\end{aligned}
$$

Then, after some manipulations one can find the Poisson bracket algebras as follows:

$$
\begin{aligned}
\{\langle\eta \widetilde{\Omega}\rangle,\langle\zeta \widetilde{\Omega}\rangle\}= & \left(\frac{\kappa^{2}}{2}\right) \widehat{\lambda}(D-2) \int d^{D} z\left[\nabla_{i} \eta \nabla_{j}\left(N^{2} \nabla^{j} \zeta\right)\right. \\
& \left.-\nabla_{i} \zeta \nabla_{j}\left(N^{2} \nabla^{j} \eta\right)\right] \mathcal{V}^{\prime}(R) N^{2} \widetilde{C}^{i} \\
& + \text { (higher-derivative contributions }),
\end{aligned}
$$

$\{\langle\eta \widetilde{\Omega}\rangle,\langle\zeta \mathcal{H}\rangle\} \approx-\int d^{D} z\left(\nabla_{i} \eta\right) \zeta N \widetilde{\Theta}^{i}$,

$\left\{\langle\eta \widetilde{\Omega}\rangle,\left\langle\zeta^{k} \mathcal{H}_{k}\right\rangle\right\}=-\int d^{D} z \zeta^{k}\left[\left(\nabla_{k} \eta\right) \widetilde{\Omega}-\left(\nabla_{k} N^{2}\right) \widetilde{C}^{i} \nabla_{i} \eta\right]$, $\{\langle\eta \widetilde{\Xi}\rangle,\langle\zeta \mathcal{H}\rangle\} \approx-\int d^{D} z\left(\nabla_{i} \eta\right) \zeta N \widetilde{\Sigma}^{i}$

+ (higher-derivative contributions),

$\left\{\langle\eta \widetilde{\Xi}\rangle,\left\langle\zeta^{k} \mathcal{H}_{k}\right\rangle\right\}=-\int d^{D} z \zeta^{k}\left[\left(\nabla_{k} \eta\right) \widetilde{\Xi}-\left(\nabla_{k} N^{2}\right) \widetilde{\Theta}^{i} \nabla_{i} \eta\right.$

$$
\left.-\nabla_{k} N\left\langle N^{2}\left(\frac{\delta \widetilde{\Theta}^{i}}{\delta N(z)}\right) \nabla_{i} \eta\right\rangle\right]
$$

+ (higher-derivative contributions)

where,

$$
\begin{aligned}
\widetilde{\Sigma}^{i}= & \left(\frac{\kappa^{2}}{2}\right) 2(D-1) N\left[\frac{(2 \lambda+1)}{(\lambda D-1)} \pi \widetilde{\Theta}^{i}-2 \pi_{k}^{i} \widetilde{\Theta}^{k}\right] \\
& +\left(\frac{\kappa^{2}}{2}\right)^{2} 2 \xi^{2}(D-1) \widehat{\lambda}\left[\nabla^{i} \mathcal{U}+\frac{2 \mathcal{V}^{i}}{(\lambda D-1)}\right] \\
& +\left(\frac{\kappa^{2}}{2}\right)^{3} \frac{8 \xi \hat{\lambda}}{\sqrt{g}} \mathcal{W}^{i}
\end{aligned}
$$$$
\mathcal{U} \equiv\left(2 N \nabla_{j} \nabla_{k} N+\nabla_{k} N \nabla_{j} N\right)\left[2 \pi^{j k}-\frac{(2 \lambda-1)}{(\lambda D-1)} \pi g^{j k}\right]
$$$$
+2 N^{2}\left[\frac{\lambda}{(\lambda D-1)} \pi R-\pi^{j k} R_{j k}\right] \text {, }
$$$$
\mathcal{V}^{i} \equiv \pi\left(-N^{2} \nabla^{i} R-\nabla^{i} N \nabla_{j} \nabla^{j} N+N \nabla^{i} \nabla^{j} \nabla_{j} N\right)
$$

$$
\begin{aligned}
+ & \nabla^{i} \pi\left[\frac{\Lambda(D+2)}{2 \xi(D-1)} N^{2}\right. \\
& \left.-\frac{(2 \lambda-D-1)}{2(D-1)}\left(N^{2} R-N \nabla_{j} \nabla^{j} N\right)\right] \\
+ & \frac{(\lambda D-1)}{(D-1)} \nabla_{j} \pi\left(N^{2} R^{i j}-N \nabla^{j} \nabla^{i} N\right) \\
& -(\lambda-1) \nabla^{i}\left(N^{2} \nabla_{j} \nabla^{j} \pi\right)-\frac{(6 \lambda-5)}{2} \nabla^{i}\left(\nabla^{j} \pi N \nabla_{j} N\right), \\
\mathcal{W}^{i} \equiv & -\frac{(2 \lambda+1)(D-2)}{2(\lambda D-1)^{2}} \pi^{3} N \nabla^{i} N \\
& -\frac{(2 \lambda+1)(D(2 \lambda+1)-3 \lambda-2)}{2(\lambda D-1)^{2}} N^{2} \pi^{2} \nabla^{i} \pi \\
+ & \frac{(D-2)}{(\lambda D-1)} \pi^{i j} \pi^{2} N \nabla_{j} N-(2 D-3) N^{2} \pi^{i k} \pi_{j k} \nabla^{j} \pi \\
+ & \frac{(4 D(2 \lambda+1)-12 \lambda-7)}{2(\lambda D-1)} N^{2} \pi^{i j} \pi \nabla_{j} \pi . \quad(\mathrm{B} 11)
\end{aligned}
$$

The Poisson algebra for the local constraints are given by

$$
\begin{aligned}
\{\widetilde{\Omega}(x), \widetilde{\Omega}(y)\}= & \left(\frac{\kappa^{2}}{2}\right) \widehat{\lambda}(D-2) \nabla_{i}^{x}\left[\mathcal{V}^{\prime}(R) N^{2} \widetilde{C}^{i}(x) \nabla_{j}^{y}\right. \\
& \left.\times\left(N^{2} \nabla_{y}^{j} \delta^{D}(x-y)\right)\right] \\
& +(\text { higher-derivative contributions }), \\
\{\widetilde{\Omega}(x), \mathcal{H}(y)\} \approx & N \widetilde{\Theta}^{i}(y) \nabla_{i}^{x} \delta^{D}(x-y), \\
\left\{\widetilde{\Omega}(x), \mathcal{H}_{k}(y)\right\}= & -\widetilde{\Omega}(y) \nabla_{k}^{y} \delta^{D}(x-y) \\
& -\widetilde{C}^{i} \nabla_{i}^{x} \delta^{D}(x-y) \nabla_{k} N^{2}(y), \\
\{\widetilde{\Xi}(x), \mathcal{H}(y)\} \approx & N \widetilde{\Sigma}^{i}(y) \nabla_{i}^{x} \delta^{D}(x-y) \\
& +(h i g h e r-\operatorname{derivative~contributions),} \\
\left\{\widetilde{\Xi}(x), \mathcal{H}_{k}(y)\right\}= & -\widetilde{\Xi}(y) \nabla_{k}^{y} \delta^{D}(x-y)-\widetilde{\Theta}^{i}(y) \nabla_{i}^{x} \delta^{D}(x \\
& -y) \nabla_{k} N^{2}(y) \\
& -\nabla_{i}^{x}\left[N^{2}(x)\left(\frac{\delta \widetilde{\Theta}^{i}(x)}{\delta N(y)}\right)\right] \nabla_{k} N(y)
\end{aligned}
$$$$
\text { +(higher-derivative contributions). (B12) }
$$

Here, we have used

$$
\begin{aligned}
\left\{\widetilde{\Omega}(x), \pi_{N}(y)\right\}= & \nabla_{i}^{x}\left[2 N \widetilde{C}^{i}(x) \delta^{D}(x-y)\right], \\
\left\{\widetilde{\Xi}(x), \pi_{N}(y)\right\}= & \nabla_{i}^{x}\left[2 N \widetilde{\Theta}^{i}(x) \delta^{D}(x-y)\right. \\
& \left.+N^{2}(x)\left(\frac{\delta \Theta^{i}(x)}{\delta N(y)}\right)\right],
\end{aligned}
$$

where

$$
\begin{aligned}
& \left(\frac{\delta \widetilde{\Theta}^{i}(x)}{\delta N(y)}\right) \\
& =-\left(\frac{\kappa^{2}}{2}\right)^{2} \frac{2 \widehat{\lambda}}{(\lambda D-1) \sqrt{g}} \mathcal{V}^{\prime}\left\{\left[(2 \lambda+1) g^{i j} \pi\right.\right. \\
& \left.\quad-2(\lambda D-1) \pi^{i j}\right] \nabla_{j} \pi(x) \delta^{D}(x-y)
\end{aligned}
$$




$$
\begin{aligned}
& \left.+\pi^{2}(x) \nabla_{x}^{i} \delta^{D}(x-y)\right\}-\left(\frac{\kappa^{2}}{2}\right) 2 \widehat{\lambda}(D-1) \\
& \times \sqrt{g} \mathcal{V}^{\prime} \nabla_{x}^{i}\left[\left(\xi R+\frac{\Lambda D}{D-1}+\frac{D-n}{D-1} \alpha R^{n}\right) \delta^{D}(x-y)\right. \\
& \left.+\nabla^{2}\left(\mathcal{V}^{\prime} \delta^{D}(x-y)\right)\right] \\
& -\left(\frac{\kappa^{2}}{2}\right)\left[2 \widehat{\lambda} \nabla_{i} \pi\left\{\mathcal{V}^{\prime}(R)(x), \mathcal{H}(y)\right\}\right. \\
& \left.+\left\{2\left(\pi^{i j}-\widehat{\lambda} g^{i j} \pi\right) \nabla_{j} \mathcal{V}^{\prime}(R)(x), \mathcal{H}(y)\right\}\right] .
\end{aligned}
$$

From (B9) or (B12), one can now compute

$$
\begin{aligned}
\left\{\widetilde{\Omega}, H_{C}\right\} & =\left\{\widetilde{\Omega},\left\langle N \mathcal{H}+N_{i} \mathcal{H}^{i}\right\rangle\right\} \\
& \approx \nabla_{i}\left[N^{2} \widetilde{\Theta}^{i}-N_{k}\left(\nabla_{k} N^{2}\right) \widetilde{C}^{i}\right]+\nabla_{k}\left(N^{k} \widetilde{\Omega}\right),
\end{aligned}
$$

$$
\begin{aligned}
\left\{\widetilde{\Xi}(x), H_{C}\right\}= & \left\{\widetilde{\Phi}(x),\left\langle N \mathcal{H}+N_{i} \mathcal{H}^{i}\right\rangle\right\} \\
\approx & \nabla_{i}\left[N^{2} \widetilde{\Sigma}^{i}-N_{k}\left(\nabla_{k} N^{2}\right) \widetilde{\Theta}^{i}\right]+\nabla_{k}\left(N^{k} \widetilde{\Xi}\right) \\
& -\nabla_{i}^{x}\left[N^{2}(x)\left\langle\left(\frac{\delta \widetilde{\Theta}^{i}(x)}{\delta N}\right) N^{k} \nabla_{k} N\right\rangle\right] \\
& + \text { (higher-derivative contributions), }
\end{aligned}
$$

which reduce to (33) and (36), respectively, for the case $\widetilde{C}^{i}=$ 0 .

\section{References}

1. P. Horava, Membranes at quantum criticality. JHEP 0903, 020 (2009). arXiv:0812.4287 [hep-th]

2. P. Hořava, Quantum gravity at a Lifshitz point. Phys. Rev. D 79, 084008 (2009). arXiv:0901.3775 [hep-th]

3. C. Charmousis, G. Niz, A. Padilla, P.M. Saffin, Strong coupling in Horava gravity. JHEP 0908, 070 (2009). arXiv:0905.2579 [hep-th]

4. M. Li, Y. Pang, A trouble with Hořava-Lifshitz gravity. JHEP 0908 , 015 (2009). arXiv:0905.2751 [hep-th]

5. D. Blas, O. Pujolas, S. Sibiryakov, On the extra mode and inconsistency of Horava gravity. JHEP 0910, 029 (2009). arXiv:0906.3046 [hep-th]

6. A. Kobakhidze, On the infrared limit of Horava's gravity with the global Hamiltonian constraint. Phys. Rev. D 82, 064011 (2010). arXiv:0906.5401 [hep-th]

7. D. Blas, O. Pujolas, S. Sibiryakov, Consistent extension of Horava gravity. Phys. Rev. Lett. 104, 181302 (2010). arXiv:0909.3525 [hep-th]

8. M.I. Park, Remarks on the scalar graviton decoupling and consistency of Horava gravity. Class. Quantum Gravity 28, 015004 (2011). arXiv:0910.1917 [hep-th]

9. K. Koyama, F. Arroja, Pathological behaviour of the scalar graviton in Hořava-Lifshitz gravity. JHEP 1003, 061 (2010). arXiv:0910.1998 [hep-th]

10. M.I. Park, Horava gravity and gravitons at a conformal point. Gen. Relativ. Gravit. 43, 2979 (2011). arXiv:0910.5117 [hep-th]

11. M. Henneaux, A. Kleinschmidt, G.L. Gomez, A dynamical inconsistency of Horava gravity. Phys. Rev. D 81, 064002 (2010). arXiv:0912.0399 [hep-th]
12. J.O. Gong, S. Koh, M. Sasaki, A complete analysis of linear cosmological perturbations in Hořava-Lifshitz gravity. Phys. Rev. D 81, 084053 (2010). arXiv:1002.1429 [hep-th]

13. J.M. Pons, P. Talavera, Remarks on the consistency of minimal deviations from General Relativity. Phys. Rev. D 82, 044011 (2010). arXiv:1003.3811 [gr-qc]

14. J. Bellorin, A. Restuccia, On the consistency of the Horava Theory. Int. J. Mod. Phys. D 21, 1250029 (2012). arXiv:1004.0055 [hep-th]

15. J. Bellorin, A. Restuccia, Closure of the algebra of constraints for a non-projectable Hořava model. Phys. Rev. D 83, 044003 (2011). arXiv:1010.5531 [hep-th]

16. R. Loll, L. Pires, Role of the extra coupling in the kinetic term in Hořava-Lifshitz gravity. Phys. Rev. D 90, 124050 (2014). arXiv:1407.1259 [hep-th]

17. P.A.M. Dirac, Generalized Hamiltonian dynamics. Can. J. Math. 2, 129 (1950)

18. R.L. Arnowitt, S. Deser, C.W. Misner, The dynamics of general relativity. Gen. Relativ. Gravit. 40, 1997 (2008). arXiv:gr-qc/0405109

19. D.O. Devecioglu, M.I. Park, in preparation

20. W. Donnelly, T. Jacobson, Hamiltonian structure of Horava gravity. Phys. Rev. D 84, 104019 (2011). arXiv:1106.2131 [hep-th]

21. J. Bellorin, A. Restuccia, Consistency of the Hamiltonian formulation of the lowest-order effective action of the complete Horava theory. Phys. Rev. D 84, 104037 (2011). arXiv:1106.5766 [hep-th]

22. E.M. Lifshitz, Zh. Eksp. Teor. Fiz. 11, 255, 269 (1941)

23. B.S. DeWitt, Quantum theory of gravity. 1. The canonical theory. Phys. Rev. 160, 1113 (1967)

24. K.S. Stelle, Renormalization of higher derivative quantum gravity. Phys. Rev. D 16, 953 (1977)

25. I.L. Buchbinder, S.L. Lyakhovich, Canonical quantization and local Measure of R**2 gravity. Class. Quantum Gravity 4, 1487 (1987)

26. I.L. Buchbinder, I.Y. Karataeva, S.L. Lyakhovich, Multidimensional $\mathrm{R} * * 2$ gravity: the structure of constraints and canonical quantization. Class. Quantum Gravity 8, 1113 (1991)

27. D.O. Devecioglu, M.I. Park, Birkhoff's theorem in Horava gravity. Phys. Rev. D 99, 104068 (2019). arXiv:1804.05698 [hep-th]

28. J. Kluson, Note aabout Hamiltonian formalism of healthy extended Horava-Lifshitz gravity. JHEP 1007, 038 (2010). arXiv: 1004.3428 [hep-th]

29. C. Lin, S. Mukohyama, A class of minimally modified gravity theories. JCAP 1710, 033 (2017). arXiv:1708.03757 [gr-qc]

30. R. Carballo-Rubio, F. Di Filippo, S. Liberati, Minimally modified theories of gravity: a playground for testing the uniqueness of general relativity. JCAP 1806, 026 (2018) [Erratum: JCAP 1811, E02 (2018)]. arXiv:1802.02537 [gr-qc]

31. S. Mukohyama, K. Noui, Minimally modified gravity: a Hamiltonian construction. JCAP 1907, 049 (2019), arXiv:1905.02000 [gr-qc]

32. X. Gao, Z.B. Yao, Spatially covariant gravity theories with two tensorial degrees of freedom: the formalism. arXiv:1910.13995 [grqc]

33. S. Shin, M.I. Park, On gauge invariant cosmological perturbations in UV-modified Hoăava gravity. JCAP 1712, 033 (2017). arXiv:1701.03844 [hep-th]

34. X. Gao, Hamiltonian analysis of spatially covariant gravity. Phys. Rev. D 90, 104033 (2014). arXiv:1409.6708 [gr-qc]

35. J. Bellorin, A. Restuccia, A. Sotomayor, Consistent Horava gravity without extra modes and equivalent to general relativity at the linearized level. Phys. Rev. D 87, 084020 (2013). arXiv:1302.1357 [hep-th]

36. E.B. Kiritsis, G. Kofinas, On Horava-Lifshitz black holes. JHEP 1001, 122 (2010). arXiv:0910.5487 [hep-th]

37. D. Capasso, A.P. Polychronakos, General static spherically symmetric solutions in Horava gravity. Phys. Rev. D 81, 084009 (2010). arXiv:0911.1535 [hep-th] 
38. A.N. Aliev, C. Senturk, Black sstrings in Hořava-Lifshitz gravity. Phys. Rev. D 84, 044010 (2011). arXiv:1106.0024 [hep-th]

39. H.W. Lee, Y.W. Kim, Y.S. Myung, Slowly rotating black holes in the Horava-Lifshitz gravity. Eur. Phys. J. C 70, 367 (2010). arXiv:1008.2243 [hep-th]

40. A.N. Aliev, C. Senturk, Slowly rotating black hole solutions to Hořava-Lifshitz gravity. Phys. Rev. D 82, 104016 (2010). arXiv: 1008.4848 [hep-th]

41. M.I. Park, The rotating black hole in renormalizable quantum gravity: the three-dimensional Hořava gravity case. Phys. Lett. B 718, 1137 (2013). arXiv:1207.4073 [hep-th]

42. M.I. Park, in preparation

43. C. Teitelboim, How commutators of constraints reflect the spacetime structure. Ann. Phys. 79, 542 (1973)

44. N. Deruelle, M. Sasaki, Y. Sendouda, D. Yamauchi, Hamiltonian formulation of (Riemann) theories of gravity. Prog. Theor. Phys. 123, 169 (2010). arXiv:0908.0679 [hep-th]

45. L.D. Faddeev, Feynman integral for singular Lagrangians. Theor. Math. Phys. 1, 1 (1969) [Teor. Mat. Fiz. 1, 3 (1969)]

46. P. Senjanovic, Path integral quantization of field theories with second class constraints. Ann. Phys. 100, 227 (1976) [Erratum: Ann. Phys. 209, 248 (1991)]

47. A.O. Barvinsky, D. Blas, M. Herrero-Valea, S.M. Sibiryakov, C.F. Steinwachs, Renormalization of Hořava gravity. Phys. Rev. D 93, 064022 (2016). arXiv:1512.02250 [hep-th]
48. A.O. Barvinsky, D. Blas, M. Herrero-Valea, S.M. Sibiryakov, C.F. Steinwachs, Hořava gravity is asymptotically free in $2+1$ dimensions. Phys. Rev. Lett. 119, 211301 (2017). arXiv:1706.06809 [hep-th]

49. A.O. Barvinsky, M. Herrero-Valea, S.M. Sibiryakov, Towards the renormalization group flow of Horava gravity in $(3+1)$ dimensions. Phys. Rev. D 100, 026012 (2019). arXiv:1905.03798 [hep-th]

50. J. Bellorin, A. Restuccia, Quantization of the Horava theory at the kinetic-conformal point. Phys. Rev. D 94, 064041 (2016). arXiv:1606.02606 [hep-th]

51. C. Teitelboim, The Hamiltonian structure of space-time. In: Held, A. (ed.) General Relativity and Gravitation, vol. 1, pp. 195-225

52. S. Das, S. Ghosh, Gauge invariant extension of linearized Horava gravity. Mod. Phys. Lett. A 26, 2793 (2011). arXiv:1104.1975 [grqc]

53. J. Bellorin, B. Droguett, Quantization of the non-projectable 2+1D Horava theory: the second-class constraints. arXiv:1912.06749 [hep-th] 